\title{
HAUSDORFF DIMENSION OF THE GRAPHS OF THE CLASSICAL WEIERSTRASS FUNCTIONS
}

\author{
WEIXIAO SHEN
}

\begin{abstract}
We show that the graph of the classical Weierstrass function $\sum_{n=0}^{\infty} \lambda^{n} \cos \left(2 \pi b^{n} x\right)$ has Hausdorff dimension $2+\log \lambda / \log b$, for every integer $b \geq 2$ and every $\lambda \in(1 / b, 1)$. Replacing $\cos (2 \pi x)$ by a general non-constant $C^{2}$ periodic function, we obtain the same result under a further assumption that $\lambda b$ is close to 1 .
\end{abstract}

\section{INTRODUCTION}

In this paper, we study the Hausdorff dimension of the graph of the following Weierstrass function

$$
W_{\lambda, b}(x)=\sum_{n=0}^{\infty} \lambda^{n} \cos \left(2 \pi b^{n} x\right), x \in \mathbb{R}
$$

where $0<\lambda<1<b$ and $b \lambda>1$. These functions, studied by Weierstrass and Hardy [6], are probably the most well-known examples of continuous but nowhere differentiable functions. Study of the graph of these and related functions from a geometric point of view as fractal sets have attracted much attention since Besicovitch and Ursell [3]. A long standing conjecture asserts that the Hausdorff dimension of the graph of $W_{\lambda, b}$ is equal to

$$
D=2+\frac{\log \lambda}{\log b}
$$

see for example [12]. Although the box dimension and packing dimension have been shown to be equal to $D$ for a large class of functions including all the functions $W_{\lambda, b}$ (see [7, 8, 14]), the conjecture about Hausdorff dimension remains open even in the case when $b$ is an integer.

Main Theorem. For any integer $b \geq 2$ and any $\lambda \in\left(b^{-1}, 1\right)$, the Hausdorff dimension of the graph of the Weierstrass function $W_{\lambda, b}$ is equal to $D$.

More generally, we consider the following function:

$$
f_{\lambda, b}^{\phi}(x)=\sum_{n=0}^{\infty} \lambda^{n} \phi\left(b^{n} x\right)
$$

where $\phi$ is a $\mathbb{Z}$-periodic function and $\lambda, b$ are as above. So $W_{\lambda, b}$ corresponds to the case $\phi(x)=\cos (2 \pi x)$. Our method also shows the following:

Date: September 25, 2018.

2010 Mathematics Subject Classification. Primary: 37C40, Secondary: 37D20, 28A80, $37 \mathrm{C} 45$. 
Theorem 1.1. For any $\mathbb{Z}$-periodic, non-constant $C^{2}$ function $\phi: \mathbb{R} \rightarrow \mathbb{R}$ and any integer $b \geq 2$ there exists $K_{0}=K_{0}(\phi, b)>1$ such that if $1<\lambda b<K_{0}$, then the graph of $f_{\lambda, b}^{\phi}$ has Hausdorff dimension $D$.

Recently, Barańsky, Bárány and Romanowska [2], based on results of Ledrappier [10] and Tsujii [16], proved that for each integer $b \geq 2$, there is a number $\lambda_{b} \in(0,1)$ such that the Hausdorff dimension of the graph of $W_{\lambda, b}$ is equal to $D$ provided that $\lambda_{b}<\lambda<1$. Furthermore, given an integer $b \geq 2$, they proved that the graph of $f_{\lambda, b}^{\phi}$ has Hausdorff dimension $D$ for generic $(\lambda, \phi)$. We refer to [2] for other progress on this and related problems. In order to prove our theorems, we have to introduce and verify a modified version of a transversality condition in [16] for all the cases. The proof of Theorem 1.1 also uses some results of [1].

The assumption that $b$ is an integer enables us to approach the problem using dynamical systems theory. Indeed, in this case, the graph of $f_{\lambda, b}^{\phi}$ can be interpreted as an invariant repeller for the expanding dynamical system $\Phi: \mathbb{R} / \mathbb{Z} \times \mathbb{R} \rightarrow \mathbb{R} / \mathbb{Z} \times \mathbb{R}$

$$
\Phi(x, y)=\left(b x \bmod 1, \frac{y-\widehat{\phi}(x)}{\lambda}\right),
$$

where $\widehat{\phi}: \mathbb{R} / \mathbb{Z} \rightarrow \mathbb{R}$ denote the map naturally induced by $\phi$. By method of ergodic theory of smooth dynamical systems, Ledrappier [10] reduced the problem on Hausdorff dimension of the graph of $f_{\lambda, b}^{\phi}$ to the study of local dimension of the measures $m_{x}$ defined below.

Let $\mathcal{A}=\{0,1, \ldots, b-1\}$, and consider the Bernoulli measure $\mathbb{P}$ on $\mathcal{A}^{\mathbb{Z}^{+}}$with uniform probabilities $\{1 / b, 1 / b, \ldots, 1 / b\}^{\mathbb{Z}^{+}}$. For $x \in \mathbb{R}$ and $\mathbf{u}=\left\{u_{n}\right\}_{n=1}^{\infty} \in$ $\mathcal{A}^{\mathbb{Z}^{+}}$, define

$$
S(x, \mathbf{u})=\sum_{n=1}^{\infty} \gamma^{n-1} \psi\left(\frac{x}{b^{n}}+\frac{u_{1}}{b^{n}}+\cdots+\frac{u_{n}}{b}\right)
$$

where

$$
\gamma=\frac{1}{\lambda b} \text { and } \psi(x)=\phi^{\prime}(x)
$$

These functions are, up to some multiplicative constant, the slope of the strong unstable manifolds of the expanding endomorphism $\Phi$. For each $x \in \mathbb{R}$, let $m_{x}$ denote the Borel probability measure in $\mathbb{R}$ obtained as pushforward of the measure $\mathbb{P}$ by the function $\mathbf{u} \mapsto S(x, \mathbf{u})$.

We say that a Borel measure $\mu$ in a metric space $X$ has local dimension $d$ at a point $x \in X$, if

$$
\lim _{r \rightarrow 0} \frac{\log \mu\left(B_{r}(x)\right)}{\log r}=d,
$$

where $B_{r}(x)$ denotes the ball of radius $r$ centered at $x$. If the local dimension of $\mu$ exists and is equal to $d$ at $\mu$-a.e. $x$, then we say that $\mu$ has local dimension $d$ and write $\operatorname{dim}(\mu)=d$. It is well-known that if $\mu$ has local dimension $d$, then any Borel set of positive measure has Haudorff dimension at least $d$. 
Ledrappier's Theorem. Let $\phi: \mathbb{R} \rightarrow \mathbb{R}$ be a continuous, piecewise $C^{1+\alpha}$ and $\mathbb{Z}$-periodic function. Assume that $\operatorname{dim}\left(m_{x}\right)=1$ holds for Lebesgue a.e. $x \in(0,1)$. Then the Hausdorff dimension of the graph of $f_{\lambda, b}^{\phi}$ is equal to $D$.

To prove this theorem, Ledrappier studied the local dimension of the measure $\mu=\mu_{\lambda, b}^{\phi}$ obtained as the lift of the Lebesgue measure on $[0,1]$ to the graph of $f_{\lambda, b}^{\phi}$. Combining results of Ledreppier and Young [11] with a variation of Marstrand's projection theorem, Ledrappier proved that $\operatorname{dim}(\mu)=D$, provided that $\operatorname{dim}\left(m_{x}\right)=1$ holds for Lebesgue almost every $x$. This proves that the Hausdorff dimension of the graph of $f_{\lambda, b}^{\phi}$ is at least $D$. As it is easy to see that the box dimension is at most $D$, the theorem follows. For the convenience of the readers not familiar with [11, we include a self-contained elementary proof of Ledrappier's Theorem in the appendix (assuming $\phi^{\prime}$ has no discontinuity for simplicity). The proof is of course motivated by the original proof in [10, but we also borrowed ideas in [9] where Keller gives an alternative proof of a weak version of Ledrappier's theorem. Keller's version is indeed enough for our purpose, although he used notation quite different from us.

Clearly, if $m_{x}$ is absolutely continuous with respect to the Lebesgue measure on $\mathbb{R}$, then $\operatorname{dim}\left(m_{x}\right)=1$. The case when $\phi(x)=d(x, \mathbb{Z})$ and $b=2$ is a famous problem in harmonic analysis and was studied first in [4. In this case, the absolute continuity of $m_{x}$ was established in [15 for almost every $\gamma \in(1 / 2,1)$. See also [13]. In general, $m_{x}$ 's are the conditional measures along vertical fibers of the unique SRB measure $\vartheta=\vartheta_{b, \gamma}^{\psi}$ of the skew product map $T: \mathbb{R} / \mathbb{Z} \times \mathbb{R} \rightarrow \mathbb{R} / \mathbb{Z} \times \mathbb{R}$,

$$
T(x, y)=(b x \bmod 1, \gamma y+\widehat{\psi}(x)),
$$

where $\psi(x)$ and $\gamma$ are as above. The map $T$ is an Anosov endomorphism and uniformly contracting along vertical fibers. The graph of the functions $x \mapsto S(x, \mathbf{u})$ are the unstable manifolds. In [16, Tsujii posed some condition on the transversality of these unstable manifolds and showed that this condition implies absolute continuity of $m_{x}$ for almost every $x$ (and the absolutely continuity of the SRB measure $\vartheta$ ). Furthermore, for given $b$, he verified his condition for generic $(\gamma, \psi)$.

However, for given $\psi$ it is not easy to verify Tsujii's condition, if possible at all. In fact, it was a major step in the recent work [2] to verify that Tsujii's condition holds for $\psi(x)=-2 \pi \sin (2 \pi x)$ when $\lambda \in\left(\lambda_{b}, 1\right)$. We shall show in Section 3 that Tsujii's condition is indeed satisfied when $b \geq 6$ for this particular $\psi$ and all $\lambda \in(1 / b, 1)$ (or equivalently, all $\gamma \in(1 / b, 1)$ ). To deal with the case $2 \leq b \leq 5$, we shall pose a modified version of Tsujii's condition. We shall show that the new (weaker) condition is still enough to guarantee absolute continuity of $m_{x}$ for Lebesgue a.e. $x$. Then we verify this new condition and conclude the proof of the Main Theorem by Ledrappier's Theorem.

Theorem 1.2. Let $b \geq 2$ be an integer, let $\gamma \in(1 / b, 1)$ and let $\psi=-2 \pi \sin (2 \pi x)$. Then the $S R B$ measure $\vartheta$ for the map $T$ is absolutely continuous with respect to the Lebesgue measure on $\mathbb{R} / \mathbb{Z} \times \mathbb{R}$ and with a square integrable density. In particular, for Lebesgue a.e. $x \in \mathbb{R}$, the measure $m_{x}$ defined above is absolutely continuous with respect to Lebesgue measure and with a square integrable density. 
In the next section, we modify Tsujii's transversality condition. In particular, we shall define a new number $\sigma(q)$ to replace the number $e(q)$ in Tsujii's work. We shall prove Theorem 1.1 and state the plan of the proof of Theorem 1.2 in that section. Sections 2-5 are devoted to the proof of Theorem 1.2 In the appendix, Section 6, we provide a proof of Ledrappier's theorem.

Acknowledgment. I would like to thank D. Feng, W. Huang and J. Wu for drawing my attention to the recent work 2. I would also like to thank H. Ruan and Y. Wang for reading carefully a first version of the manuscript and pointing out a number of errors.

\section{TSUJII'S TRANSVERSALITY CONDITION ON FAT SOLENOIDAL ATTRACTORS}

In this section, we study a map $T$ of the form (1.3), where $b \geq 2$ is an integer, $b^{-1}<\gamma<1$ and $\psi$ is a $\mathbb{Z}$-periodic $C^{1}$ function. These maps were studied in [16] from measure-theoretical point of view, and in [1 from topological point of view. In [16], Section 2, it was shown that $T$ has a unique SRB measure $\vartheta$, for which Lebesgue almost every point $(x, y)$ in $\mathbb{R} / \mathbb{Z} \times \mathbb{R}$ is a generic point, i.e.

$$
\frac{1}{n} \sum_{i=0}^{n-1} \delta_{T^{i}(x, y)} \rightarrow \vartheta \text { as } n \rightarrow \infty,
$$

in the weak star topology, where $\delta$. denote the Dirac measure. The measure $\vartheta$ has an explicit expression through the measures $m_{x}$ defined in the introduction: identifying $\mathbb{R} / \mathbb{Z}$ with $[0,1)$ in the natural way, for each Borel set $B \subset \mathbb{R} / \mathbb{Z} \times \mathbb{R}$,

$$
\vartheta(B)=\int_{0}^{1} m_{x}\left(B_{x}\right) d x
$$

where $B_{x}=\{y \in \mathbb{R}:(x, y) \in B\}$. We are interested in the absolute continuity of the SRB measure $\vartheta$, or equivalently, the absolute continuity of $m_{x}$ for Lebesgue almost every $x$. In [16], Tsujii posed some condition on the transversality of the graphs of the functions $S(x, \mathbf{u})$ (which are understood as unstable manifolds of $T$ ) which guarantees the absolute continuity of $\vartheta$.

In this section, we introduce a modified version of Tsujii's condition and show that the weaker condition already implies absolute continuity of $\vartheta$. We shall prove Theorem 1.1 by verifying the modified condition.

Notation. For each $x \in \mathbb{R}$ and $\left(u_{1} u_{2} \cdots u_{q}\right) \in \mathcal{A}^{q}$, let

$$
x(\mathbf{u})=\frac{x+u_{1}+u_{2} b+\cdots+u_{q} b^{q-1}}{b^{q}} .
$$

We use $S^{\prime}(x, \mathbf{u})$ to denote the derivative of $S(x, \mathbf{u})$ regarded as a function of $x$.

2.1. Transversality. We say that two words $\mathbf{i}, \mathbf{j} \in \mathcal{A}^{Z^{+}}$are $(\varepsilon, \delta)$-transversal at a point $x_{0} \in \mathbb{R}$ if one of the following holds:

$$
\left|S\left(x_{0}, \mathbf{i}\right)-S\left(x_{0}, \mathbf{j}\right)\right|>\varepsilon \text { or }\left|S^{\prime}\left(x_{0}, \mathbf{i}\right)-S^{\prime}\left(x_{0}, \mathbf{j}\right)\right|>\delta .
$$

Otherwise, we say that $\mathbf{i}$ and $\mathbf{j}$ are $(\varepsilon, \delta)$-tangent at $x_{0}$. Let $E\left(q, x_{0} ; \varepsilon, \delta\right)$ denote the set of pairs $(\mathbf{k}, \mathbf{l}) \in \mathcal{A}^{q} \times \mathcal{A}^{q}$ for which there exist $\mathbf{u}, \mathbf{v} \in \mathcal{A}^{\mathbb{Z}^{+}}$such that $\mathbf{k u}$ and $\mathbf{l v}$ are $(\varepsilon, \delta)$-tangent at $x_{0}$. Let

$$
E\left(q, x_{0}\right)=\bigcap_{\varepsilon>0} \bigcap_{\delta>0} E\left(q, x_{0} ; \varepsilon, \delta\right)
$$


and

$$
e\left(q, x_{0}\right)=\max _{\mathbf{k} \in \mathcal{A}^{q}} \#\left\{\mathbf{l} \in \mathcal{A}^{q}:(\mathbf{k}, \mathbf{l}) \in E\left(q, x_{0}\right)\right\} .
$$

For $J \subset \mathbb{R}$, define

and

$$
\begin{gathered}
E(q, J ; \varepsilon, \delta)=\bigcup_{x_{0} \in J} E\left(q, x_{0} ; \varepsilon, \delta\right), \\
E(q, J)=\bigcap_{\varepsilon>0} \bigcap_{\delta>0} E(q, J ; \varepsilon, \delta)
\end{gathered}
$$

$$
e(q, J)=\max _{\mathbf{k} \in \mathcal{A}^{q}} \#\left\{\mathbf{l} \in \mathcal{A}^{q}:(\mathbf{k}, \mathbf{l}) \in E(q, J)\right\} .
$$

Tsujii's notation $e(q)$ is defined as

$$
e(q)=\lim _{p \rightarrow \infty} \max _{k=0}^{b^{p}-1} e\left(q,\left[\frac{k}{b^{p}}, \frac{k+1}{b^{p}}\right]\right) .
$$

The following was proved in [16, see Proposition 8 in Section 4.

Theorem 2.1 (Tsujii). If there exists a positive integer $q$ such that $e(q)<$ $(\gamma b)^{q}$, then the SRB measure $\vartheta$ is absolutely continuous with respect to the Lebesgue measure on $\mathbb{R} / \mathbb{Z} \times \mathbb{R}$ with square integrable density. In particular, for Lebesgue a.e. $x \in[0,1), m_{x}$ is absolutely continuous with respect to the Lebesgue measure on $\mathbb{R}$ and with square integrable density.

Remark. It is obvious that $e(q) \geq e\left(q, x_{0}\right)$ for all $x_{0} \in[0,1)$. Indeed, by Porposition 2.2 and Lemma 2.3. one can prove $e(q)=\max _{x \in[0,1)} e(q, x)=$ $\max _{x \in \mathbb{R}} e(q, x)$, although we do not need this fact.

We are going to define $\sigma(q)$. Let us say that a measurable function $\omega$ : $[0,1) \rightarrow(0, \infty)$ is a weight function if $\|\omega\|_{\infty}<\infty$ and $\|1 / \omega\|_{\infty}<\infty$. A testing function of order $q$ is a measurable function $V:[0,1) \times \mathcal{A}^{q} \times \mathcal{A}^{q} \rightarrow[0, \infty)$. A testing function of order $q$ is called admissible if there exist $\varepsilon>0$ and $\delta>0$ such that the following hold: For any $x \in[0,1)$, if $(\mathbf{u}, \mathbf{v}) \in E(q, x ; \varepsilon, \delta)$, then

$$
V(x, \mathbf{u}, \mathbf{v}) V(x, \mathbf{v}, \mathbf{u}) \geq 1 .
$$

So in particular, we have $V(x, \mathbf{u}, \mathbf{u}) \geq 1$ for each $x \in[0,1)$ and each $\mathbf{u} \in \mathcal{A}^{q}$.

Given a weight function $\omega$ and an admissible testing function $V$ of order $q$, define a new measurable function $\Sigma_{V, \omega}^{q}:[0,1) \rightarrow \mathbb{R}$ as follows: For each $x \in[0,1)$, let

$$
\Sigma_{V, \omega}^{q}(x)=\sup \left\{\frac{\omega(x)}{\omega(x(\mathbf{u}))} \sum_{\mathbf{v} \in \mathcal{A}^{q}} V(x, \mathbf{u}, \mathbf{v}): \mathbf{u} \in \mathcal{A}^{q}\right\} .
$$

Define

$$
\sigma(q)=\inf \left\|\Sigma_{V, \omega}^{q}\right\|_{\infty}
$$

where the infimum is taken over all weight functions $\omega$ and admissible testing functions $V$ of order $q$. In $\S 2.2$, we shall prove the following theorem:

Theorem 2.2. If there exists an integer $q \geq 1$ such that $\sigma(q)<(\gamma b)^{q}$ then the $S R B$ measure $\vartheta$ is absolutely continuous with respect to the Lebesgue measure on $\mathbb{R} / \mathbb{Z} \times \mathbb{R}$ with square integrable density. In particular, for Lebesgue a.e. $x \in[0,1), m_{x}$ is absolutely continuous with respect to the Lebesgue measure on $\mathbb{R}$ and with square integrable density. 
The parameter $\sigma(q)$ takes into account the fact that the number

$$
\#\{\mathbf{v}:(\mathbf{u}, \mathbf{v}) \in E(q, x ; \varepsilon, \delta)\}
$$

may depend on $x$ and $\mathbf{u}$ in a significant way. On the other hand, the parameter $e(q)$ is the supremum of such numbers over all possible choices of $x$ and $\mathbf{u}$.

Lemma 2.1. $\sigma(q) \leq e(q)$.

Proof. Fix $\varepsilon, \delta>0$. Let $\omega=1$ be the constant weight function. For each $x \in[0,1)$, define

$$
V(x, \mathbf{u}, \mathbf{v})= \begin{cases}1, & \text { if }(\mathbf{u}, \mathbf{v}) \in E(q, x ; \varepsilon, \delta) \\ 0, & \text { otherwise }\end{cases}
$$

Then for any $x \in[0,1)$ and $\mathbf{u} \in \mathcal{A}^{q}$, we have

$$
\frac{\omega(x)}{\omega(x(\mathbf{u}))} \sum_{\mathbf{v}} V(x, \mathbf{u}, \mathbf{v})=\#\{\mathbf{v}:(\mathbf{u}, \mathbf{v}) \in E(q, x ; \varepsilon, \delta)\} .
$$

Thus

$$
\sigma(q) \leq\left\|\Sigma_{V, \omega}\right\|_{\infty} \leq \sup _{x \in[0,1), \mathbf{u} \in \mathcal{A}^{q}} \#\{\mathbf{v}: \mathbf{u}, \mathbf{v} \in E(q, x ; \varepsilon, \delta)\} .
$$

Letting $\varepsilon, \delta \rightarrow 0$, we obtain $\sigma(q) \leq e(q)$.

The following proposition collects a few facts about the quantifiers in the transversality conditions.

Proposition 2.2. For $\boldsymbol{k}, \boldsymbol{l} \in \mathcal{A}^{q}$, the following hold:

(1) For any $x_{0} \in \mathbb{R},(\boldsymbol{k}, \boldsymbol{l}) \in E\left(q, x_{0}\right)$ if and only if there exist $\boldsymbol{u}$ and $\boldsymbol{v}$ in $\mathcal{A}^{\mathbb{Z}^{+}}$such that $S(x, \boldsymbol{k u})-S(x, \boldsymbol{l v})$ has a multiple zero at $x_{0}$.

(2) If $(\boldsymbol{k}, \boldsymbol{l}) \notin E\left(q, x_{0}\right)$, then there is a neighborhood $U$ of $x_{0}$ and $\varepsilon, \delta>0$, such that $(\boldsymbol{k}, \boldsymbol{l}) \notin E(q, U ; \varepsilon, \delta)$.

(3) For any compact $K \subset \mathbb{R}$, if $(\boldsymbol{k}, \boldsymbol{l}) \notin E(q, K)$, then there exist $\varepsilon, \delta>0$ such that $(\boldsymbol{k}, \boldsymbol{l}) \notin E(q, K ; \varepsilon, \delta)$.

(4) For any $\varepsilon>\varepsilon^{\prime}>0, \delta>\delta^{\prime}>0$ there exists $\eta>0$ such that if $\left|x-x_{0}\right|<$ $\eta,(\boldsymbol{k}, \boldsymbol{l}) \notin E\left(q, x_{0} ; \varepsilon, \delta\right)$ then $(\boldsymbol{k}, \boldsymbol{l}) \notin E\left(q, x ; \varepsilon^{\prime}, \delta^{\prime}\right)$.

Proof. Let us endow $\mathcal{A}^{\mathbb{Z}^{+}}$with the usual product topology of the discrete topology on $\mathcal{A}$. Then $\mathcal{A}^{\mathbb{Z}^{+}}$is compact. Moreover, if $\mathbf{u}^{n} \rightarrow \mathbf{u}$ in $\mathcal{A}^{\mathbb{Z}^{+}}$, then

$$
S\left(x, \mathbf{u}^{n}\right) \rightarrow S(x, \mathbf{u}) \text { and } S^{\prime}\left(x, \mathbf{u}^{n}\right) \rightarrow S^{\prime}(x, \mathbf{u})
$$

uniformly as $n \rightarrow \infty$.

(1) The "if" part is obvious. For the "only if" part, assume $(\mathbf{k}, \mathbf{l}) \in E\left(q, x_{0}\right)$. Then for any $n=1,2, \ldots,(\mathbf{k}, \mathbf{l}) \in E\left(q, x_{0} ; 1 / n, 1 / n\right)$, and so there exist $\mathbf{u}^{n}, \mathbf{v}^{n} \in$ $\mathcal{A}^{\mathbb{Z}^{+}}$such that

$$
\left|S\left(x_{0}, \mathbf{k u}^{n}\right)-S\left(x_{0}, \mathbf{l} \mathbf{v}^{n}\right)\right| \leq 1 / n, \text { and }\left|S^{\prime}\left(x_{0}, \mathbf{k u}^{n}\right)-S^{\prime}\left(x_{0}, \mathbf{l} \mathbf{v}^{n}\right)\right| \leq 1 / n .
$$

After passing to a subsequence, we may assume $\mathbf{u}^{n} \rightarrow \mathbf{u}$ and $\mathbf{v}^{n} \rightarrow \mathbf{v}$ in $\mathcal{A}^{\mathbb{Z}^{+}}$ as $n \rightarrow \infty$. Then

$$
S\left(x_{0}, \mathbf{k u}\right)-S\left(x_{0}, \mathbf{l v}\right)=S^{\prime}\left(x_{0}, \mathbf{k u}\right)-S^{\prime}\left(x_{0}, \mathbf{l v}\right)=0 .
$$


(2) Arguing by contradiction, assume that the statement is false. Then there exists $\left\{x_{n}\right\}_{n=1}^{\infty}$ such that $x_{n} \rightarrow x_{0}$ and $(\mathbf{k}, \mathbf{l}) \in E\left(q, x_{n} ; 1 / n, 1 / n\right)$. Thus there exist $\mathbf{u}^{n}, \mathbf{v}^{n} \in \mathcal{A}^{\mathbb{Z}^{+}}$such that

$$
\left|S\left(x_{n}, \mathbf{k u}^{n}\right)-S\left(x_{n}, \mathbf{l} \mathbf{v}^{n}\right)\right| \leq 1 / n \text {, and }\left|S^{\prime}\left(x_{n}, \mathbf{k} \mathbf{u}^{n}\right)-S^{\prime}\left(x_{n}, \mathbf{l} \mathbf{v}^{n}\right)\right| \leq 1 / n .
$$

After passing to a subsequence we may assume $\mathbf{u}^{n} \rightarrow \mathbf{u}, \mathbf{v}^{n} \rightarrow \mathbf{v}$. It follows that

$$
S\left(x_{0}, \mathbf{k u}\right)-S\left(x_{0}, \mathbf{l v}\right)=S^{\prime}\left(x_{0}, \mathbf{k u}\right)-S^{\prime}\left(x_{0}, \mathbf{l v}\right)=0,
$$

a contradiction.

(3) follows from (2).

(4) Since $\psi$ is $\mathbb{Z}$-periodic and $C^{1}$, for any $\xi>0$ there exists $\eta>0$ such that if $\left|x_{1}-x_{2}\right|<\eta$, then $\left|\psi\left(x_{1}\right)-\psi\left(x_{2}\right)\right|<\xi$ and $\left|\psi^{\prime}\left(x_{1}\right)-\psi^{\prime}\left(x_{2}\right)\right|<\xi$. Then for any $\mathbf{u} \in \mathcal{A}^{\mathbb{Z}^{+}}$, we have

$$
\begin{gathered}
\left|S\left(x_{1}, \mathbf{u}\right)-S\left(x_{2}, \mathbf{u}\right)\right| \leq \xi /(1-\gamma), \\
\left|S^{\prime}\left(x_{1}, \mathbf{u}\right)-S^{\prime}\left(x_{2}, \mathbf{u}\right)\right| \leq \xi /(b-\gamma) .
\end{gathered}
$$

The statement follows.

We shall also use the following symmetry of the functions $S(x, \mathbf{u})$.

Lemma 2.3. For any $\boldsymbol{u} \in \mathcal{A}^{\mathbb{Z}^{+}}, x \in \mathbb{R}$ and $q \in \mathbb{Z}^{+}$, we have $e(q, x+1)=$ $e(q, x)$ and $m_{x+1}=m_{x}$.

Proof. Indeed, for any $\mathbf{u} \in \mathcal{A}^{\mathbb{Z}^{+}}$and $x \in \mathbb{R}$, we have

$$
S(x+1, \mathbf{u})=S(x, \operatorname{add}(\mathbf{u})),
$$

where add : $\mathcal{A}^{\mathbb{Z}^{+}} \rightarrow \mathcal{A}^{\mathbb{Z}^{+}}$the adding machine which can be defined as follows: Given $\mathbf{u}=\left\{u_{n}\right\}_{n=1}^{\infty} \in \mathcal{A}^{\mathbb{Z}^{+}}$, defining inductively $v_{n}, w_{n} \in \mathcal{A}$ with the following properties:

- $w_{1}=1$;

- If $u_{n}+w_{n}<b$ then $v_{n}=u_{n}+w_{n}$ and $w_{n+1}=0$; otherwise, define $v_{n}=0$ and $w_{n}=1$,

then $\operatorname{add}(\mathbf{u})=\left\{v_{n}\right\}_{n=1}^{\infty}$. This is a homeomorphism of $\mathcal{A}^{\mathbb{Z}^{+}}$which preserves the Bernoulli measure $\mathbb{P}$. Thus $m_{x+1}=m_{x}$.

Since the first $q$ elements of $\operatorname{add}(\mathbf{u})$ depend only on the first $q$ element of $\mathbf{u}$, add induces a bijection from $\mathcal{A}^{q}$ onto itself, denoted also by add. By definition, $(\mathbf{k}, \mathbf{l}) \in E(q, x+1)$ if and only if $(\operatorname{add}(\mathbf{k}), \operatorname{add}(\mathbf{l})) \in E(q, x)$. Thus $e(q, x+1)=e(q, x)$.

2.2. Proof of Theorem 2.2. The proof of Theorem 2.2 is an easy modification of Tsujii's proof of Theorem 2.1. Fix a weight function $\omega$ and an admissible testing function $V$ of order $q$ such that

$$
\left\|\Sigma_{V, \omega}\right\|_{\infty}<(\gamma b)^{q} .
$$

By definition, there exist $\varepsilon, \delta>0$ such that for any $x \in[0,1)$, if $(\mathbf{u}, \mathbf{v}) \in$ $E(q, x ; \varepsilon, \delta)$, then

$$
V(x, \mathbf{u}, \mathbf{v}) V(x, \mathbf{v}, \mathbf{u}) \geq 1 .
$$

For Borel measures $\rho$ and $\rho^{\prime}$ on $\mathbb{R}$ any $r>0$, let

$$
\left(\rho, \rho^{\prime}\right)_{r}=\int_{\mathbb{R}} \rho(B(y, r)) \rho^{\prime}(B(y, r)) d y,
$$


and let

$$
\|\rho\|_{r}=\sqrt{(\rho, \rho)_{r}} .
$$

For a Borel subset $J \subset \mathbb{R}$, define

$$
I_{r}(J)=\frac{1}{r^{2}} \int_{J} \omega(x)\left\|m_{x}\right\|_{r}^{2} d x
$$

and write $I_{r}=I_{r}([0,1))$.

According to Lemma 4 of [16], $\liminf _{r \rightarrow 0}\|\rho\|_{r}<\infty$ implies that $\rho$ is absolutely continuous with respect to the Lebesgue measure and the density function is square integrable. Consequently, if $\lim \inf I_{r}<\infty$, then the conclusion of the theorem holds.

With slight abuse of language, let $T^{q}\left(m_{x}\right)$ denote the pushforward of the measure $m_{x}$ under the map $y \mapsto \pi_{2} \circ T^{q}(x, y)$, where $\pi_{2}(x, y)=y$. Then

$$
m_{x}=\frac{1}{b^{q}} \sum_{\mathbf{i} \in \mathcal{A}^{q}} T^{q}\left(m_{x(\mathbf{i})}\right) .
$$

Thus

$$
\left\|m_{x}\right\|_{r}^{2}=b^{-2 q} \sum_{\mathbf{i}, \mathbf{j}}\left(T^{q} m_{x(\mathbf{i})}, T^{q} m_{x(\mathbf{j})}\right)_{r} .
$$

Let

$$
I_{r}^{o}(J)=\frac{1}{b^{2 q} r^{2}} \int_{J} \omega(x) \sum_{(\mathbf{i}, \mathbf{j}) \notin E(q, x ; \varepsilon, \delta)}\left(T^{q}\left(m_{x(\mathbf{i})}\right), T^{q}\left(m_{x(\mathbf{j})}\right)\right)_{r} d x
$$

and

$$
I_{r}^{*}(J)=\frac{1}{b^{2 q} r^{2}} \int_{J} \omega(x) \sum_{(\mathbf{i}, \mathbf{j}) \in E(q, x ; \varepsilon, \delta)}\left(T^{q}\left(m_{x(\mathbf{i})}\right), T^{q}\left(m_{x(\mathbf{j})}\right)\right)_{r} d x .
$$

Then

$$
I_{r}(J)=I_{r}^{o}(J)+I_{r}^{*}(J) .
$$

We shall also write $I_{r}^{o}=I_{r}^{0}([0,1))$ and $I_{r}^{*}=I_{r}^{*}([0,1))$.

Lemma 2.4. There exists $C>0$ such that $I_{r}^{o} \leq C$ holds for all $r>0$.

Proof. Fix $\varepsilon^{\prime} \in(0, \varepsilon)$ and $\delta^{\prime} \in(0, \delta)$. By Proposition 2.2 (4), there exists a positive integer $p$, such that if $x \in J_{p, k}:=\left[k / b^{p},(k+1) / b^{p}\right]$ and $(\mathbf{i}, \mathbf{j}) \notin$ $E(q, x ; \varepsilon, \delta)$, then $(\mathbf{i}, \mathbf{j}) \notin E\left(q, J_{p, k} ; \varepsilon^{\prime}, \delta^{\prime}\right)$. It follows that

$$
I_{r}^{o} \leq \frac{\|\omega\|_{\infty}}{b^{2 q} r^{2}} \sum_{k=0}^{b^{p}-1} \int_{J_{k, p}} \sum_{(\mathbf{i}, \mathbf{j}) \notin E\left(q, J_{p, k} ; \varepsilon^{\prime}, \delta^{\prime}\right)}\left(T^{q}\left(m_{x(\mathbf{i})}\right), T^{q}\left(m_{x(\mathbf{j})}\right)\right)_{r} d x .
$$

In Proposition 6 in [16, it was proved that there exists $C^{\prime}=C^{\prime}\left(p, \varepsilon^{\prime}, \delta^{\prime}\right)>0$ such that if $(\mathbf{i}, \mathbf{j}) \notin E\left(q, J_{p, k} ; \varepsilon^{\prime}, \delta^{\prime}\right)$, then

$$
\int_{J_{p, k}}\left(T^{q}\left(m_{x(\mathbf{i})}\right), T^{q}\left(m_{x(\mathbf{j})}\right)\right)_{r} d x \leq C^{\prime} r^{2} .
$$

Thus $I_{r}^{o} \leq C$.

In order to estimate the terms $I_{r}^{*}(J)$, Tsujii observed

Lemma 2.5. For any $i \in \mathcal{A}^{q}$ and any $x \in \mathbb{R}$, we have

$$
\left\|T^{q}\left(m_{x(\boldsymbol{i})}\right)\right\|_{r}^{2}=\gamma^{q}\left\|m_{x(\boldsymbol{i})}\right\|_{\gamma^{-q_{r}}}^{2} .
$$


Proof. This follows immediately from the fact that $T^{q}$ is a contraction of rate $\gamma^{q}$ in the vertical direction.

Lemma 2.6. For each $r>0$, we have

$$
I_{r}^{*} \leq \frac{\left\|\Sigma_{V, \omega}\right\|_{\infty}}{(b \gamma)^{q}} I_{\gamma^{-q_{r}}} .
$$

Proof. Let us first prove that for each $x \in[0,1)$,

$$
\sum_{(\mathbf{u}, \mathbf{v}) \in E(q, x ; \varepsilon, \delta)}\left(T^{q} m_{x(\mathbf{u})}, T^{q} m_{x(\mathbf{v})}\right)_{r} \leq \gamma^{q} \sum_{\mathbf{u} \in \mathcal{A}^{q}}\left(\sum_{\mathbf{v} \in \mathcal{A}^{q}} V(x, \mathbf{u}, \mathbf{v})\right)\left\|m_{x(\mathbf{u})}\right\|_{r / \gamma^{q}}^{2} .
$$

To this end, let $\mathbf{u}_{k}, k=1,2, \ldots, b^{q}$ be all the elements of $\mathcal{A}^{q}$. Fix $x \in[0,1)$ and prepare the following notation: $V_{k l}=V\left(x, \mathbf{u}_{k}, \mathbf{u}_{l}\right), x_{k}=x\left(\mathbf{u}_{k}\right)$ and

$$
\theta_{k l}= \begin{cases}1 & \text { if }\left(\mathbf{u}_{k}, \mathbf{u}_{l}\right) \in E(q, x ; \varepsilon, \delta) \\ 0 & \text { otherwise. }\end{cases}
$$

Then

$$
\sum_{(\mathbf{u}, \mathbf{v}) \in E(q, x ; \varepsilon, \delta)}\left(T^{q} m_{x(\mathbf{u})}, T^{q} m_{x(\mathbf{v})}\right)_{r}=\sum_{k=1}^{b^{q}}\left\|T^{q} m_{x_{k}}\right\|_{r}^{2}+2 \sum_{1 \leq k<l \leq b^{q}} \theta_{k l}\left(T^{q} m_{x_{k}}, T^{q} m_{x_{l}}\right)_{r} .
$$

For each $1 \leq k<l \leq b^{q}$, by the Cauchy-Schwarz inequality,

$$
\left(T^{q} m_{x_{k}}, T^{q} m_{x_{l}}\right)_{r} \leq\left\|T^{q} m_{x_{k}}\right\|_{r}\left\|T^{q} m_{x_{l}}\right\|_{r} .
$$

Thus

$$
2 \theta_{k l}\left(T^{q} m_{x_{k}}, T^{q} m_{x_{l}}\right)_{r} \leq V_{k l}\left\|T^{q} m_{x_{k}}\right\|_{r}^{2}+V_{l k}\left\|T^{q} m_{x_{l}}\right\|_{r}^{2} .
$$

Indeed, this is trivial if $\theta_{k l}=0$, while if $\theta_{k l}=1$, it follows from the previous inequality and $V_{k l} V_{l k} \geq 1$. Consequently,

$$
\begin{aligned}
2 \sum_{1 \leq k<l \leq b^{q}} \theta_{k l}\left(T^{q} m_{x_{k}}, T^{q} m_{x_{l}}\right)_{r} & \leq \sum_{1 \leq k<l \leq b^{q}}\left(V_{k l}\left\|T_{q} m_{x_{k}}\right\|_{r}^{2}+V_{l k}\left\|T^{q} m_{x_{l}}\right\|_{r}^{2}\right) \\
& =\sum_{k=1}^{b^{q}}\left(\sum_{\substack{1 \leq l \leq b^{q} \\
l \neq k}} V_{k l}\right)\left\|T^{q} m_{x_{k}}\right\|_{r}^{2},
\end{aligned}
$$

and hence

$$
\sum_{(\mathbf{u}, \mathbf{v}) \in E(q, x ; \varepsilon, \delta)}\left(T^{q} m_{x(\mathbf{u})}, T^{q} m_{x(\mathbf{v})}\right)_{r} \leq \sum_{k=1}^{b^{q}}\left(\sum_{l=1}^{b^{q}} V_{k l}\right)\left\|T^{q} m_{x_{k}}\right\|_{r}^{2} .
$$

By Lemma 2.5 the inequality (2.1) follows.

Multiplying $\omega(x)$ on both sides of (2.1), we obtain

$$
\omega(x) \sum_{(\mathbf{u}, \mathbf{v}) \in E(q, x ; \varepsilon, \delta)}\left(T^{q} m_{x(\mathbf{u})}, T^{q} m_{x(\mathbf{v})}\right)_{r} \leq \gamma^{q} \sum_{\mathbf{u} \in \mathcal{A}^{q}} \Sigma_{V, \omega}(x) \omega(x(\mathbf{u}))\left\|m_{x(\mathbf{u})}\right\|_{\gamma^{-q_{r}}}^{2} .
$$

Dividing both side by $b^{2 q} r^{2}$ and integrating over $[0,1)$, we obtain

$$
I_{r}^{*} \leq \frac{\left\|\Sigma_{V, \omega}\right\|_{\infty}}{b^{2 q} \gamma^{q}} \frac{1}{\left(\gamma^{-q} r\right)^{2}} \sum_{\mathbf{u} \in \mathcal{A}^{q}} \int_{0}^{1}\left\|m_{x(\mathbf{u})}\right\|_{\gamma^{-q} r}^{2} \omega(x(\mathbf{u})) d x .
$$


Let $J(\mathbf{u})=\{x(\mathbf{u}): 0 \leq x<1\}$. Then

$$
\int_{0}^{1}\left\|m_{x(\mathbf{u})}\right\|_{\gamma^{-q_{r}}}^{2} \omega(x(\mathbf{u})) d x=b^{q} \int_{J(\mathbf{u})}\left\|m_{x}\right\|_{\gamma^{-q_{r}}}^{2} \omega(x) d x .
$$

Since $J(\mathbf{u}), \mathbf{u} \in \mathcal{A}^{q}$, form a partition of $[0,1)$, it follows that

$$
I_{r}^{*} \leq \frac{\left\|\Sigma_{V, \omega}\right\|_{\infty}}{(\gamma b)^{q}} \frac{1}{\left(\gamma^{-q} r\right)^{2}} \int_{0}^{1}\left\|m_{x}\right\|_{\gamma^{-q} r}^{2} \omega(x) d x=\frac{\left\|\Sigma_{V, \omega}\right\|_{\infty}}{(\gamma b)^{q}} I_{\gamma^{-q} r} .
$$

Completion of proof of Theorem 2.2, By Lemma 2.4 and Lemma 2.6, there exists a constant $C>0$ such that

$$
I_{r}=I_{r}^{o}+I_{r}^{*} \leq C+\beta I_{\gamma^{-q_{r}}},
$$

holds for all $r>0$, where $\beta=\left\|\Sigma_{V, \omega}\right\|_{\infty} /(\gamma b)^{q} \in(0,1)$. As $I_{r}<\infty$ for each $r>0$, it follows that $\liminf _{r \searrow 0} I_{r}<\infty$. By the remarks at the beginning of this subsection, the conclusion of the theorem follows.

2.3. Proof of Theorem 1.1, In this subsection, we shall prove Theorem 1.1 using Theorem 2.2,

Lemma 2.7. Suppose that for each $x \in[0,1), E(q, x) \neq \mathcal{A}^{q} \times \mathcal{A}^{q}$. Then

$$
\sigma(q) \leq b^{q}-2+2 / \alpha,
$$

where $\alpha=\alpha(b, q)>1$ satisfies

$$
2-\alpha=\left(b^{q}-2\right) \alpha(\alpha-1) .
$$

Proof. By Lemma 2.3. the assumption implies that for each $x \in \mathbb{R}, E(q, x) \neq$ $\mathcal{A}^{q} \times \mathcal{A}^{q}$. By Proposition 2.2 (2) and compactness of [0,1], there exists $\varepsilon>$ $0, \delta>0$ such that $E(q, x ; \varepsilon, \delta) \neq \mathcal{A}^{q} \times \mathcal{A}^{q}$ for each $x \in[0,1]$. So we can find measurable functions $\mathbf{k}, \mathbf{l}:[0,1) \rightarrow \mathcal{A}^{q}$ such that $(\mathbf{k}(x), \mathbf{l}(x)) \notin E(q, x ; \varepsilon, \delta)$. Define $\omega(x)=1$ for all $x \in[0,1)$. Define

$$
V(x, \mathbf{u}, \mathbf{v})= \begin{cases}1 & \text { if } \mathbf{u}, \mathbf{v} \notin\{\mathbf{k}(x), \mathbf{l}(x)\} \text { or } \mathbf{u}=\mathbf{v} ; \\ 0 & \text { if }(\mathbf{u}, \mathbf{v})=(\mathbf{k}(x), \mathbf{l}(x)) \text { or }(\mathbf{l}(x), \mathbf{k}(x)) ; \\ \alpha & \text { if } \mathbf{u} \in\{\mathbf{k}(x), \mathbf{l}(x)\} \text { but } \mathbf{v} \notin\{\mathbf{k}(x), \mathbf{l}(x)\} ; \\ \alpha^{-1} & \text { if } \mathbf{u} \notin\{\mathbf{k}(x), \mathbf{l}(x)\} \text { but } \mathbf{v} \in\{\mathbf{k}(x), \mathbf{l}(x)\} .\end{cases}
$$

Then $V$ is an admissible test function of order $q$. For every $x \in[0,1)$, if $\mathbf{u} \notin\{\mathbf{k}(x), \mathbf{l}(x)\}$, then

$$
\frac{\omega(x)}{\omega(x(\mathbf{u}))} \sum_{\mathbf{v} \in \mathcal{A}^{q}} V(x, \mathbf{u}, \mathbf{v})=b^{q}-2+\frac{2}{\alpha},
$$

and if $\mathbf{u} \in\{\mathbf{k}(x), \mathbf{l}(x)\}$, then

$$
\frac{\omega(x)}{\omega(x(\mathbf{u}))} \sum_{\mathbf{v} \in \mathcal{A}^{q}} V(x, \mathbf{u}, \mathbf{v})=1+\left(b^{q}-2\right) \alpha=b^{q}-2+\frac{2}{\alpha} .
$$

Thus $\sigma(q) \leq\left\|\Sigma_{V, \omega}\right\|_{\infty} \leq b^{q}-2+2 / \alpha$.

We shall use some results obtained in [1]. Fix an integer $b \geq 2$. We say that a $\mathbb{Z}$-periodic continuous function $\psi: \mathbb{R} \rightarrow \mathbb{R}$ is cohomologous to 0 if there exists a continuous $\mathbb{Z}$-periodic function $f: \mathbb{R} \rightarrow \mathbb{R}$ such that $\psi(x)=f(b x)-f(x)$ holds for all $x \in \mathbb{R}$. The main step is the following lemma. 
Lemma 2.8. Assume that $\psi: \mathbb{R} \rightarrow \mathbb{R}$ is a $\mathbb{Z}$-periodic $C^{1}$ function that is not cohomologous to zero and $\int_{0}^{1} \psi(x) d x=0$. Then there exists $\gamma_{1} \in(0,1)$ and a positive integer $N$ such that if $\gamma_{1}<\gamma<1$, then $E(N, x) \neq \mathcal{A}^{N} \times \mathcal{A}^{N}$ for each $x \in \mathbb{R}$.

Proof. We shall prove that there exists $\gamma_{1}, N_{1}$ and $x_{1} \in \mathbb{R}$ such that $E\left(N_{1}, x_{1}\right) \neq$ $\mathcal{A}^{N_{1}} \times \mathcal{A}^{N_{1}}$. Note that this is enough for the conclusion of this lemma. Indeed, let $\mathbf{k}, \mathbf{l} \in \mathcal{A}^{N_{1}}$ be such that $(\mathbf{k}, \mathbf{l}) \notin E\left(N_{1}, x_{1}\right)$. Then by Proposition 2.2 (2), there exist $\varepsilon>0$ and $\delta>0$ a neighborhood $U$ of $x_{1}$ such that $(\mathbf{k}, \mathbf{l}) \notin E\left(N_{1}, x_{1} ; \varepsilon, \delta\right)$. Let $N_{2}$ be a positive integer such that $b^{N_{2}} U+\mathbb{Z}=\mathbb{R}$. Then for any $y \in \mathbb{R}$, there exists $x \in U$ and $k \in \mathbb{Z}$ such that $y=b^{N_{2}} x+k$. Since the words $(00 \cdots 0 \mathbf{k}),(00 \cdots 0 \mathbf{l}) \in \mathcal{A}^{N_{2}+N_{1}}$ are transversal at $b^{N_{2}} x$, we have $E\left(N_{1}+N_{2}, y-k\right) \neq \mathcal{A}^{N_{1}+N_{2}} \times \mathcal{A}^{N_{1}+N_{2}}$ which is equivalent to $E\left(N_{1}+N_{2}, y\right) \neq$ $\mathcal{A}^{N_{1}+N_{2}} \times \mathcal{A}^{N_{1}+N_{2}}$ by Lemma 2.3 .

For each $\mathbf{u} \in \mathcal{A}^{\mathbb{Z}^{+}}$, let $G: \mathbb{R} \rightarrow \mathbb{R}$ be defined as

$$
G(x, \mathbf{u})=\sum_{n=1}^{\infty} \frac{1}{b^{n}} \psi^{\prime}\left(\frac{x+u_{1}+u_{2} b+\cdots+u_{n} b^{n-1}}{b^{n}}\right) .
$$

Note that $G(x)=G(x, \mathbf{0})$ satisfies the functional equation

$$
b G(b x)=\psi^{\prime}(x)+G(x) .
$$

We claim that $G(x)$ is not $\mathbb{Z}$-periodic. Indeed, otherwise, from the equation above, we obtain $\int_{0}^{1} G(x) d x=0$. Then $g(x)=\int_{0}^{x} G(t) d t$ defines a $\mathbb{Z}$-periodic function. Since $\psi^{\prime}=b g^{\prime}(b x)-g^{\prime}(x)$ and

$$
\int_{0}^{1} \psi(x) d x=\int_{0}^{1}(g(b x)-g(x)) d x=0,
$$

it follows that $\psi(x)=g(b x)-g(x)$ holds for all $x$. This contradicts the assumption that $\psi$ is not cohomologous to zero.

Since $G(x+1)=G(x,(100 \cdots))$, it follows that there exists $x_{1} \in[0,1)$ such that

$$
5 \delta:=\left|G\left(x_{1},(000 \cdots)\right)-G\left(x_{1},(100 \cdots)\right)\right|>0 .
$$

Let $C=\max _{x \in[0,1]}\left|\psi^{\prime}(x)\right|$. Let $N_{1}$ be a positive integer such that

$$
2 C<\delta b^{N_{1}}(b-1)
$$

and let $\gamma_{1} \in(0,1)$ be such that

$$
\left(1-\gamma_{1}^{N_{1}}\right) C<\delta(b-1)
$$

Then, for any $\mathbf{k}, \mathbf{l} \in \mathcal{A}^{\mathbb{Z}^{+}}$with $k_{1}=k_{2}=\cdots=k_{N_{1}}=0, l_{1}=1, l_{2}=l_{3}=\cdots=$ $l_{N_{1}}=0$, we have

$$
\begin{aligned}
\left|\frac{d}{d x} S\left(x_{1}, \mathbf{k}\right)-G\left(x_{1}\right)\right| \leq & \sum_{n=1}^{N_{1}} \frac{\left(1-\gamma^{n-1}\right)}{b^{n}} C+2 C \sum_{n=N_{1}+1}^{\infty} b^{-n} \\
& <\left(1-\gamma_{1}^{N_{1}}\right) C(b-1)^{-1}+2 C\left((b-1) b^{N_{1}}\right)^{-1}<2 \delta,
\end{aligned}
$$

and similarly,

$$
\left|\frac{d}{d x} S\left(x_{1}, \mathbf{l}\right)-G\left(x_{1}+1\right)\right|<2 \delta .
$$


Therefore,

$$
\left|\frac{d}{d x} S\left(x_{1}, \mathbf{k}\right)-\frac{d}{d x} S\left(x_{1}, \mathbf{l}\right)\right| \geq \delta .
$$

It follows that the two words $(00 \cdots 0),(10 \cdots 0) \in \mathcal{A}^{N_{1}}$ are transversal at $x_{1}$, hence

$$
E\left(N_{1}, x_{1}\right) \neq \mathcal{A}^{N_{1}} \times \mathcal{A}^{N_{1}} .
$$

Proof of Theorem 1.1. Let $\psi=\phi^{\prime}$, so $\psi$ is a $\mathbb{Z}$-periodic non-constant $C^{1}$ function and $\int_{0}^{1} \psi(x) d x=0$. Consider the map $T$ as in (1.3). By Ledrappier's Theorem, it suffices to prove the measures $m_{x}$ defined for this map $T$ are absolutely continuous for Lebesgue almost every $x \in[0,1]$.

First we assume that $\psi$ is not cohomologous to 0 . By Lemma 2.8, there exists $\gamma_{1} \in(0,1)$ and $N$ such that if $\gamma_{1}<\gamma<1$, then $E(N, x) \neq \mathcal{A}^{N} \times \mathcal{A}^{N}$ for each $x \in \mathbb{R}$. By Lemma 2.7, this implies that $\sigma(N)<b^{N}-2+\frac{2}{\alpha}$ where $\alpha=\alpha(b, N) \in(1,2)$. Thus there exists $\gamma_{0} \in\left(\gamma_{1}, 1\right)$ such that if $\gamma>\gamma_{0}$ then $\sigma(N)<(b \gamma)^{N}$. By Theorem 2.2, it follows that $m_{x}$ is absolutely continuous for Lebesgue a.e. $x \in[0,1]$.

To complete the proof, we shall use a few results of [1. Assume that $\psi$ is cohomologous to 0 and let $\psi_{1}: \mathbb{R} \rightarrow \mathbb{R}$ be a $\mathbb{Z}$-periodic continuous function such that $\psi_{1}(b x)-\psi_{1}(x)=\psi(x)$. By Lemma $5.2(5)$ and Lemma 5.8 (2) of that paper, $\psi_{1}$ is $C^{1}$, and $T_{b, \gamma, \psi}$ is $C^{1}$ conjugate to $T_{b, \gamma, \psi_{1}}$. By adding a constant if necessary, we may assume $\int_{0}^{1} \psi_{1}(x) d x=0$. If $\psi_{1}$ is not cohomologous to zero, then we are done. Otherwise, repeat the argument. By Lemma 5.6 of that paper, any $\mathbb{Z}$-periodic non-constant $C^{1}$ function $\psi$ is not infinitely cohomologous to zero. Thus the procedure stops within finitely many steps.

2.4. Plan of Proof of Theorem 1.2. Theorem 1.2 follows from the following theorem by Theorem 2.2 .

Theorem 2.3. For an integer $b \geq 2,1 / b<\gamma<1$ and $\psi(x)=-2 \pi \sin (2 \pi x)$, consider the map $T$ as in (1.3). Then there exists a positive integer $q$ such that $\sigma(q)<(b \gamma)^{q}$.

The rest of the paper is devoted to the proof of Theorem 2.3. The proof uses special property of the map $\psi$ and breaks into several cases.

Proof of Theorem 2.3. The case $b \geq 6$ is proved in Theorem 3.1 (i). The case $b=5$ is proved in Theorem 4.1. The case $b=4$ is proved in Theorem 4.2, The case $b=3$ is proved in Theorem 4.3. The case $b=2$ follows from Corollary 5.13 and Proposition 5.16

To conclude this section, we include a few lemmas which will be used in later sections. The first lemma is about a new symmetric property of the functions $S(x, \mathbf{u})$ in the case that $\psi$ is odd.

Lemma 2.9 (Symmetry). Assume that $\psi(x)$ is an odd function. Then for any $\boldsymbol{i}=\left\{i_{n}\right\}_{n=1}^{\infty} \in \mathcal{A}^{\mathbb{Z}^{+}}$, letting $\boldsymbol{i}^{\prime}=\left\{i_{n}^{\prime}\right\}_{n=1}^{\infty}$ with $i_{n}^{\prime}=b-1-i_{n}$, we have

$$
-S(x, \boldsymbol{i})=S\left(1-x, \boldsymbol{i}^{\prime}\right) .
$$

Proof. This follows from the definition of $S(\cdot, \cdot)$. 
The next three lemmas will be used to obtain upper bounds for $\sigma(q)$.

Lemma 2.10. Let $q \geq 1$ be an integer. Suppose that there are constants $\varepsilon>0$ and $\delta>0$ and $K \subset[0,1)$ with the following properties:

(i) For $x \in K, e(q, x ; \varepsilon, \delta)=1$ and for $x \in[0,1) \backslash K, e(q, x ; \varepsilon, \delta) \leq 2$;

(ii) If $(\boldsymbol{u}, \boldsymbol{v}) \in E(q, x ; \varepsilon, \delta)$ for some $x \in[0,1) \backslash K$ and $\boldsymbol{u} \neq \boldsymbol{v}$, then both $x(\boldsymbol{u})$ and $x(\boldsymbol{v})$ belong to $K$.

Then $\sigma(q) \leq \sqrt{2}$.

Proof. We define suitable weight function $\omega$ and testing function $V$. Let $L=$ $[0,1) \backslash K$. Define

$$
\omega(x)= \begin{cases}\sqrt{2} & \text { if } x \in K \\ 1 & \text { if } x \in L .\end{cases}
$$

Define

$$
V(x, \mathbf{u}, \mathbf{v})= \begin{cases}1 & \text { if }(\mathbf{u}, \mathbf{v}) \in E(q, x ; \varepsilon, \delta) \\ 0 & \text { otherwise }\end{cases}
$$

Then for $x \in K$ and any $\mathbf{u} \in \mathcal{A}^{q}$,

$$
\frac{\omega(x)}{\omega(x(\mathbf{u}))} \sum_{\mathbf{v}} V(x, \mathbf{u}, \mathbf{v})=\frac{\omega(x)}{\omega(x(\mathbf{u}))} \leq \sqrt{2} .
$$

For $x \in L$ and $\mathbf{u} \in \mathcal{A}^{q}$, if $\mathbf{u}$ is not $(\varepsilon, \delta)$-tangent to any other element of $\mathcal{A}^{q}$ at $x$, then we have

$$
\frac{\omega(x)}{\omega(x(\mathbf{u}))} \sum_{\mathbf{v}} V(x, \mathbf{u}, \mathbf{v})=\frac{\omega(x)}{\omega(x(\mathbf{u}))} \leq 1 ;
$$

otherwise, we have $\omega(x)=1$ and $\omega(x(\mathbf{u}))=\sqrt{2}$

$$
\frac{\omega(x)}{\omega(x(\mathbf{u}))} \sum_{\mathbf{v}} V(x, \mathbf{u}, \mathbf{v})=\frac{\omega(x)}{\omega(x(\mathbf{u}))} \cdot 2 \leq \sqrt{2} .
$$

It follows that $\sigma(q) \leq \Sigma_{V, w} \leq \sqrt{2}$.

Lemma 2.11. Let $q \geq 1$ be an integer. Suppose that there are constants $\varepsilon>0$ and $\delta>0$ and $K \subset[0,1)$ with the following properties:

(i) For $x \in K, e(q, x ; \varepsilon, \delta) \leq 1$ and for $x \in[0,1) \backslash K, e(q, x: \varepsilon, \delta) \leq 2$;

(ii) If $(\boldsymbol{u}, \boldsymbol{v}) \in E(q, x ; \varepsilon, \delta)$ for some $x \in[0,1) \backslash K$ and $\boldsymbol{u} \neq \boldsymbol{v}$, then either $x(\boldsymbol{u}) \in K$ or $x(\boldsymbol{v}) \in K$.

Then $\sigma(q) \leq(\sqrt{5}+1) / 2$.

Proof. Let $L=[0,1) \backslash K$. Define

$$
\omega(x)= \begin{cases}(\sqrt{5}+1) / 2 & \text { if } x \in K \\ 1 & \text { otherwise }\end{cases}
$$

For $x \in K$, define

$$
V(x, \mathbf{u}, \mathbf{v})= \begin{cases}1 & \text { if } \mathbf{u}=\mathbf{v} \\ 0 & \text { otherwise }\end{cases}
$$

For $x \in L$, define

$$
V(x, \mathbf{u}, \mathbf{v})= \begin{cases}0 & \text { if }(\mathbf{u}, \mathbf{v}) \notin E(q, x ; \varepsilon, \delta) ; \\ 1 & \text { if } \mathbf{u}=\mathbf{v} ; \\ (\sqrt{5}+1) / 2 & \text { if }(\mathbf{u}, \mathbf{v}) \in E(q, x ; \varepsilon, \delta), \mathbf{u} \neq \mathbf{v}, \text { and } x(\mathbf{u}) \in K \\ (\sqrt{5}-1) / 2 & \text { if }(\mathbf{u}, \mathbf{v}) \in E(q, x ; \varepsilon, \delta), \mathbf{u} \neq \mathbf{v}, \text { and } x(\mathbf{u}) \notin K\end{cases}
$$


Then for $x \in K$ and any $\mathbf{u} \in \mathcal{A}^{q}$, we have

$$
\frac{\omega(x)}{\omega(x(\mathbf{u}))} \sum_{\mathbf{v}} V(x, \mathbf{u}, \mathbf{v}) \leq \frac{\sqrt{5}+1}{2} .
$$

For $x \in L$ and $\mathbf{u} \in \mathcal{A}^{q}$, if $x(\mathbf{u}) \in K$, we have

$$
\frac{\omega(x)}{\omega(x(\mathbf{u}))} \sum_{\mathbf{v}} V(x, \mathbf{u}, \mathbf{v}) \leq \frac{1}{(\sqrt{5}+1) / 2}\left(\frac{\sqrt{5}+1}{2}+1\right)=\frac{\sqrt{5}+1}{2} ;
$$

if $x(\mathbf{u}) \notin K$, then

$$
\frac{\omega(x)}{\omega(x(\mathbf{u}))} \sum_{\mathbf{v}} V(x, \mathbf{u}, \mathbf{v}) \leq \frac{1}{1}\left(\frac{\sqrt{5}-1}{2}+1\right)=\frac{\sqrt{5}+1}{2} .
$$

In conclusion, we have $\sigma(q) \leq\left\|\Sigma_{V, \omega}\right\|_{\infty} \leq(\sqrt{5}+1) / 2$.

The next lemma is more technical and will only be used in the case $b=2$.

Lemma 2.12. Let $q \geq 1$ be an integer. Suppose there are three pairwise disjoint subsets $K_{0}, K_{1}, K_{2}$ of $[0,1)$ with $K_{0} \cup K_{1} \cup K_{2}=[0,1)$ and constants $\varepsilon, \delta>0$ such that the following hold:

(i) For each $x \in K_{0}, e(q, x ; \varepsilon, \delta)=1$;

(ii) For each $x \in K_{1}$, there exist $\boldsymbol{a}_{x}, \boldsymbol{b}_{x} \in \mathcal{A}^{q}$ such that $x\left(\boldsymbol{a}_{x}\right), x\left(\boldsymbol{b}_{x}\right) \in K_{0}$ and such that $\left(\boldsymbol{a}_{x}, \boldsymbol{b}_{x}\right)$ and $\left(\boldsymbol{b}_{x}, \boldsymbol{a}_{x}\right)$ are the only possible non-trivial element of $E(q, x ; \varepsilon, \delta)$;

(iii) For $x \in K_{2}$, there exist $\boldsymbol{a}_{x}, \boldsymbol{b}_{x}, \boldsymbol{c}_{x} \in \mathcal{A}^{q}$ such that $x\left(\boldsymbol{a}_{x}\right), x\left(\boldsymbol{b}_{x}\right) \in K_{0}$ and $x\left(\boldsymbol{c}_{x}\right) \in K_{1}$ and such that $\left(\boldsymbol{a}_{x}, \boldsymbol{b}_{x}\right),\left(\boldsymbol{a}_{x}, \boldsymbol{c}_{x}\right),\left(\boldsymbol{b}_{x}, \boldsymbol{a}_{x}\right)$ and $\left(\boldsymbol{c}_{x}, \boldsymbol{a}_{x}\right)$ are the only possible non-trivial elements of $E(q, x ; \varepsilon, \delta)$.

Then

$$
\sigma(q) \leq t<1.61
$$

where $t>\sqrt{2}$ is the unique solution of the following equation

$$
\frac{1}{t^{2}-1}+\frac{2}{t^{3}-2}+1=t^{2}
$$

Proof. Let $s=t^{2} / 2$. Note that $t>s>1$. Define

$$
\omega(x)= \begin{cases}t & \text { if } x \in K_{0} \\ s & \text { if } x \in K_{1} \\ 1 & \text { if } x \in K_{2}\end{cases}
$$

For $x \in K_{0} \cup K_{1}$, define

$$
V(x, \mathbf{u}, \mathbf{v})= \begin{cases}0 & \text { if }(\mathbf{u}, \mathbf{v}) \notin E(q, x ; \varepsilon, \delta) \\ 1 & \text { otherwise }\end{cases}
$$

For $x \in K_{2}$, define

$$
V(x, \mathbf{u}, \mathbf{v})= \begin{cases}1 & \text { if } \mathbf{u}=\mathbf{v} \\ t s-1 & \text { if }(\mathbf{u}, \mathbf{v})=\left(\mathbf{c}_{x}, \mathbf{a}_{x}\right) \\ (t s-1)^{-1} & \text { if }(\mathbf{u}, \mathbf{v})=\left(\mathbf{a}_{x}, \mathbf{c}_{x}\right) \\ t^{2}-1 & \text { if }(\mathbf{u}, \mathbf{v})=\left(\mathbf{b}_{x}, \mathbf{a}_{x}\right) \\ \left(t^{2}-1\right)^{-1} & \text { if }(\mathbf{u}, \mathbf{v})=\left(\mathbf{a}_{x}, \mathbf{b}_{x}\right) \\ 0 & \text { otherwise. }\end{cases}
$$


Then for $x \in K_{0}$, and any $\mathbf{u} \in \mathcal{A}^{q}$ we have

$$
\frac{\omega(x)}{\omega(x(\mathbf{u}))} \sum_{\mathbf{v} \in \mathcal{A}^{q}} V(x, \mathbf{u}, \mathbf{v}) \leq t
$$

for $x \in K_{1}, \mathbf{u} \in\left\{\mathbf{a}_{x}, \mathbf{b}_{x}\right\}$,

$$
\frac{\omega(x)}{\omega(x(\mathbf{u}))} \sum_{\mathbf{v} \in \mathcal{A}^{q}} V(x, \mathbf{u}, \mathbf{v})=2 s / t=t
$$

for $x \in K_{1}, \mathbf{u} \notin\left\{\mathbf{a}_{x}, \mathbf{b}_{x}\right\}$,

$$
\frac{\omega(x)}{\omega(x(\mathbf{u}))} \sum_{\mathbf{v} \in \mathcal{A}^{q}} V(x, \mathbf{u}, \mathbf{v}) \leq s<t
$$

for $x \in K_{2}$,

$$
\frac{\omega(x)}{\omega\left(x\left(\mathbf{a}_{x}\right)\right)} \sum_{\mathbf{v} \in \mathcal{A}^{q}} V(x, \mathbf{u}, \mathbf{v})=\frac{1}{t}\left(\frac{1}{t^{2}-1}+\frac{1}{t s-1}+1\right)=t
$$

for $x \in K_{2}$,

$$
\frac{\omega(x)}{\omega\left(x\left(\mathbf{b}_{x}\right)\right)} \sum_{\mathbf{v} \in \mathcal{A}^{q}} V\left(x, \mathbf{b}_{x}, \mathbf{v}\right)=\frac{1}{t}\left(1+t^{2}-1\right)=t
$$

for $x \in K_{2}$,

$$
\frac{\omega(x)}{\omega\left(x\left(\mathbf{c}_{x}\right)\right)} \sum_{\mathbf{v} \in \mathcal{A}^{q}} V\left(x, \mathbf{c}_{x}, \mathbf{v}\right)=\frac{1}{s}(1+t s-1)=t ;
$$

and for $x \in K_{2}, \mathbf{u} \notin\left\{\mathbf{a}_{x}, \mathbf{b}_{x}, \mathbf{c}_{x}\right\}$,

$$
\frac{\omega(x)}{\omega(x(\mathbf{u}))} \sum_{\mathbf{v} \in \mathcal{A}^{q}} V(x, \mathbf{u}, \mathbf{v}) \leq 1
$$

Therefore

$$
\sigma(q) \leq\left\|\Sigma_{V, \omega}(x)\right\|_{\infty} \leq t
$$

\section{The CASE When $b$ IS LARGE}

In this and next sections, we shall prove Theorem 2.3. So we consider a map $T$ of the form (1.3) with $\psi=-2 \pi \sin (2 \pi x)$. The main result of this section is the following:

Theorem 3.1. (1) If $b \geq 6$, then $\sigma(1) \leq e(1)<\gamma b$.

(2) If $b=4,5$, then either $e(1)=2$ or $e(1)<\gamma b$.

(3) If $b=3$, then either $e(1)=2$ or $\sigma(1)<\gamma b$.

We start with a few lemmas. Let

$$
\Delta_{b, \gamma}=\max _{t \in \mathbb{R}}(\sin (b t)+\gamma \sin (t)) .
$$

Besides the trivial bound: $\Delta_{b, \gamma} \leq 1+\gamma$, we also need the following:

Lemma 3.1. For each $\gamma \in(0,1)$, we have

$$
\begin{aligned}
& \Delta_{6, \gamma} \leq \max (1+0.972 \gamma, 0.99+\gamma) \\
& \Delta_{3, \gamma} \leq 1+0.71 \gamma .
\end{aligned}
$$


Proof. Let us first prove (3.2). Indeed, if $\sin t \leq 0.71$ then the inequality holds. So assume $\sin t>0.71$. Then $\sin (3 t)=3 \sin t-4 \sin ^{3} t \leq 0.71$, and hence $\sin (3 t)+\gamma \sin t \leq 0.71+\gamma \leq 1+0.71 \gamma$.

Let us prove (3.1). If $\sin t \leq 0.972$ then the inequality holds. So assume $\sin t>0.972$, then $\sin (3 t) \leq 3 \cdot 0.972-4 \cdot 0.972^{3}=-0.757$. Then

$$
|\sin (6 t)| \leq 2|\sin (3 t)| \sqrt{1-\sin ^{2}(3 t)}<0.99 .
$$

Lemma 3.2. If $(k, l) \in E\left(1, x^{*}\right)$, then

$$
\begin{gathered}
\left|\sin \frac{2 \pi\left(x^{*}+k\right)}{b}-\sin \frac{2 \pi\left(x^{*}+l\right)}{b}\right| \leq \frac{2 \Delta_{b, \gamma} \gamma}{1-\gamma^{2}} \leq \frac{2 \gamma}{1-\gamma} \\
\left|\cos \frac{2 \pi\left(x^{*}+k\right)}{b}-\cos \frac{2 \pi\left(x^{*}+l\right)}{b}\right| \leq \frac{2 \gamma}{b-\gamma}, \\
4 \sin ^{2} \frac{\pi(k-l)}{b} \leq\left(\frac{2 \gamma \Delta_{b, \gamma}}{1-\gamma^{2}}\right)^{2}+\left(\frac{2 \gamma}{b-\gamma}\right)^{2} \leq\left(\frac{2 \gamma}{1-\gamma}\right)^{2}+\left(\frac{2 \gamma}{b-\gamma}\right)^{2} .
\end{gathered}
$$

Proof. By Proposition $2.2(1)$, there exists $\mathbf{k}=\left\{k_{n}\right\}_{n=1}^{\infty}$ and $\mathbf{l}=\left\{l_{n}\right\}_{n=1}^{\infty}$ in $\mathcal{A}^{\mathbb{Z}^{+}}$with $k_{1}=k$ and $l_{1}=l$ such that for $F(x):=-(2 \pi)^{-1}(S(x, \mathbf{k})-S(x, \mathbf{l}))$, we have $F\left(x^{*}\right)=F^{\prime}\left(x^{*}\right)=0$. Let $f(x)=\sin (2 \pi b x)+\gamma \sin (2 \pi x)$. Then

$$
\left|-\frac{S(x, \mathbf{k})}{2 \pi}-\sin \frac{2 \pi(x+k)}{b}\right| \leq \sum_{n=1}^{\infty} \gamma^{2 n-1}\left|f\left(x_{2 n+1}\right)\right| \leq \frac{2 \gamma \Delta_{b, \gamma}}{1-\gamma^{2}},
$$

and

$$
\left|-\frac{b S^{\prime}(x, \mathbf{k})}{4 \pi^{2}}-\cos \frac{2 \pi(x+k)}{b}\right| \leq \sum_{n=2}^{\infty}\left(\frac{\gamma}{b}\right)^{n-1}\left|\cos \left(2 \pi x_{n}\right)\right| \leq \frac{\gamma}{b-\gamma},
$$

where $x_{n}=\left(x+k_{1}+k_{2} b+\cdots+k_{n} b^{n-1}\right) / b^{n}$. Similarly,

$$
\left|-\frac{S(x, \mathbf{l})}{2 \pi}-\sin \frac{2 \pi(x+l)}{b}\right| \leq \frac{2 \gamma \Delta_{b, \gamma}}{1-\gamma^{2}}
$$

and

Therefore,

$$
\left|-\frac{b S^{\prime}(x, \mathbf{l})}{4 \pi^{2}}-\cos \frac{2 \pi(x+l)}{b}\right| \leq \frac{\gamma}{b-\gamma}
$$

$$
\begin{aligned}
& \left|F(x)-\left(\sin \frac{2 \pi(x+k)}{b}-\sin \frac{2 \pi(x+l)}{b}\right)\right| \leq \frac{2 \gamma \Delta_{b, \gamma}}{1-\gamma^{2}}, \\
& \left|\frac{b F^{\prime}(x)}{2 \pi}-\left(\cos \frac{2 \pi(x+k)}{b}-\cos \frac{2 \pi(x+l)}{b}\right)\right| \leq \frac{2 \gamma}{b-\gamma} .
\end{aligned}
$$

Substituting $x=x^{*}$ gives us (3.3) and (3.4). The inequality (3.5) follows from these two inequalities and the following

$$
(\cos x-\cos y)^{2}+(\sin x-\sin y)^{2}=4 \sin ^{2} \frac{y-x}{2} .
$$


Pick up $z \in[0,1]$ such that $e(1, z)=e(1)$ and pick up $k \in\{0,1, \ldots, b-1\}$ such that

$$
\#\{l \in\{0,1, \ldots, b-1\}:(k, l) \in E(1, z)\}=e(1) .
$$

Let $k_{1}, k_{2}, \ldots, k_{e(1)}$ be all the elements in $E(1, z)$, arranged in such a way that

$$
\sin \left(2 \pi x_{1}\right) \leq \sin \left(2 \pi x_{2}\right) \leq \cdots \leq \sin \left(2 \pi x_{e(1)}\right)
$$

where $x_{i}=\left(z+k_{i}\right) / b$.

Lemma 3.3. Under the above circumstances, the following holds:

(1) For each $1 \leq i<j \leq e(1)$, we have

$$
\left|\sin \left(2 \pi x_{i}\right)-\sin \left(2 \pi x_{j}\right)\right| \geq \frac{2 \theta_{1}(b, \gamma)}{b},
$$

where

$$
\theta_{1}(b, \gamma)=\sqrt{\max \left(0,\left(b \sin \frac{\pi}{b}\right)^{2}-\frac{4 \gamma^{2} b^{2}}{(b-\gamma)^{2}}\right)} .
$$

(2) If $k_{i}=k$ or $k_{j}=k$, then

$$
\left|\sin \left(2 \pi x_{i}\right)-\sin \left(2 \pi x_{j}\right)\right| \geq \frac{2 \theta_{0}(b, \gamma)}{b},
$$

where

$$
\theta_{0}(b, \gamma)=\sqrt{\max \left(0,\left(b \sin \frac{\pi}{b}\right)^{2}-\frac{\gamma^{2} b^{2}}{(b-\gamma)^{2}}\right)} .
$$

(3) If $k_{i}-k_{j} \neq \pm 1 \bmod b$, then

$$
\left|\sin \left(2 \pi x_{i}\right)-\sin \left(2 \pi x_{j}\right)\right| \geq \frac{2 \theta_{2}(b, \gamma)}{b},
$$

where

$$
\theta_{2}(b, \gamma)=\sqrt{\max \left(0,\left(b \sin \frac{2 \pi}{b}\right)^{2}-\frac{4 \gamma^{2} b^{2}}{(b-\gamma)^{2}}\right)} .
$$

Proof. For each $1 \leq i<j \leq e(1)$, we have

$\left|\cos \left(2 \pi x_{i}\right)-\cos \left(2 \pi x_{j}\right)\right|^{2}+\left|\sin \left(2 \pi x_{i}\right)-\sin \left(2 \pi x_{j}\right)\right|^{2}=4 \sin ^{2}\left(\pi\left(x_{i}-x_{j}\right)\right) \geq 4 \sin ^{2} \frac{\pi}{b}$.

If $k_{i}=k$ or $k_{j}=k$ then by (3.4), the inequality (3.8) follows.

In general, from (3.4), we obtain

$$
\left|\cos \left(2 \pi x_{i}\right)-\cos \left(2 \pi x_{j}\right)\right| \leq \frac{4 \gamma}{b-\gamma},
$$

which, together with (3.12), implies (3.6).

If $k_{i}-k_{j} \neq \pm 1 \bmod b$, then

$$
\left|\cos \left(2 \pi x_{i}\right)-\cos \left(2 \pi x_{j}\right)\right|^{2}+\left|\sin \left(2 \pi x_{i}\right)-\sin \left(2 \pi x_{j}\right)\right|^{2} \geq 4 \sin ^{2} \frac{2 \pi}{b},
$$

which, together with (3.13), implies (3.10). 
WEIXIAO SHEN

3.1. The case when $b \geq 6$. We shall prove Theorem 3.1 in the case $b \geq 6$. We separate the argument in two propositions.

Proposition 3.4. Assume $b \geq 6$. If there exists $1 \leq i<e(1)$ such that $k_{i+1}-k_{i} \neq \pm 1 \bmod b$, then $e(1)<\gamma b$.

Proof. Under assumption, we have

$$
\begin{aligned}
\frac{2 \theta_{2}(b, \gamma)}{b}+(e(1)-2) \frac{2 \theta_{1}(b, \gamma)}{b} \leq \sum_{i=1}^{e(1)-1}\left(\sin \left(2 \pi x_{i+1}\right)-\sin \left(2 \pi x_{i}\right)\right) \\
=\sin \left(2 \pi x_{e(1)}\right)-\sin \left(2 \pi x_{1}\right) \leq 2
\end{aligned}
$$

and so

$$
b \geq(e(1)-2) \theta_{1}(\gamma, b)+\theta_{2}(\gamma, b)
$$

We may assume

$$
\gamma \leq \frac{b-\theta_{2}(b, \gamma)+2 \theta_{1}(b, \gamma)}{\theta_{1}(b, \gamma) b}
$$

for otherwise we are done.

Note that $t \mapsto \sin t / t$ is monotone decreasing in $\left[0, \frac{\pi}{2}\right)$. Since $b \geq 6$, we have

$$
\theta_{1}(6, \gamma) \geq \theta_{1}(6,1)=1.8 \text { and } \theta_{2}(6, \gamma) \geq \theta_{2}(6,1)=\sqrt{21.24}>4 .
$$

By (3.16), it follows that

Therefore, we have

$$
\gamma \leq \frac{b-4+2 \cdot 1.8}{1.8 b}<\frac{5}{9}
$$

$$
\theta_{1}(6, \gamma) \geq \theta_{1}(6,5 / 9)>2.5 \text { and } \theta_{2}(6, \gamma) \geq \theta_{2}(6,5 / 9)>5,
$$

and hence

$$
\gamma \leq \frac{b-5+2 \cdot 2.5}{2.5 b}=\frac{2}{5}
$$

Moreover, by (3.15),

$$
b>2.5 e(1)
$$

Case 1. $e(1) \leq 3$.

Indeed, this is clear if $e(1)=1$ as we assume $\gamma b>1$. If $e(1)=2$ or 3 , then there exists $i, j$ such that $k_{i}=k \neq k_{j}$, and

$$
\left|\sin \left(2 \pi x_{i}\right)-\sin \left(2 \pi x_{j}\right)\right| \geq \frac{2 \theta_{2}(b, \gamma)}{b} .
$$

On the other hand, since $\left(k_{i}, k_{j}\right) \in E(1, z)$, we have

$$
\left|\sin \left(2 \pi x_{i}\right)-\sin \left(2 \pi x_{j}\right)\right| \leq \frac{2 \gamma}{1-\gamma} .
$$

Therefore,

$$
\frac{2 \gamma}{1-\gamma} \geq \frac{2 \theta_{2}(b, \gamma)}{b}
$$

which, together with (3.17) and (3.18), implies that

$$
\gamma b \geq(1-\gamma) \theta_{2}(b, \gamma)>5(1-2 / 5)=3 \geq e(1) .
$$

Case 2. $e(1) \geq 4$. 
By (3.19), we have $b>2.5 e(1) \geq 10$. Since $b$ is an integer, this implies $b \geq 11$. Thus $\theta_{1}(b, \gamma) \geq \theta_{1}(11,2 / 5)>2.9$ and $\theta_{2}(b, \gamma) \geq \theta_{2}(11,2 / 5)>5.8$, where we use the numerics: $\sin (\pi / 11)>0.28$ and $\sin (2 \pi / 11)>0.54$. By (3.15) and (3.16), we obtain

$$
b \geq 2.9 e(1) \text { and } \gamma \leq \frac{10}{29} .
$$

Let us first consider the case $e(1)=4$. Then by (3.20), we have $b \geq 12$, hence $\theta_{1}(b, \gamma) \geq \theta_{1}(12,10 / 29)>3$. On the other hand, there exists $1 \leq i, j \leq 4$ such that $k_{i}=k$ and $|i-j| \geq 2$. Thus

$$
\frac{2 \gamma}{1-\gamma} \geq\left|\sin \left(2 \pi x_{i}\right)-\sin \left(2 \pi x_{j}\right)\right| \geq \frac{4 \theta_{1}(\gamma, b)}{b} .
$$

Therefore, $\frac{2 \gamma}{1-\gamma}>\frac{12}{b}$, which implies that

$$
\gamma b>6(1-\gamma)
$$

If $\gamma \leq 1 / 3$, then it follows that $\gamma b>4=e(1)$. If $\gamma>1 / 3$, then $\gamma b>12 \cdot(1 / 3)=$ $4=e(1)$.

Now let us assume $e(1) \geq 5$. Then by $(3.20)$ we have $b>2.9 e(1) \geq 14.5$ which implies that $b \geq 15$. Then

$$
\theta_{1}(\gamma, b) \geq \theta_{1}(15,10 / 29)>3 \text { and } \theta_{2}(\gamma, b) \geq \theta_{2}(15,10 / 29)>6 .
$$

By (3.16), it follows that $\gamma<1 / 3$. Since

$$
\frac{4 \gamma}{1-\gamma} \geq\left|\sin \left(2 \pi x_{e(1)}\right)-\sin \left(2 \pi x_{1}\right)\right| \geq \frac{2 \theta_{1}}{b}(e(1)-2)+\frac{2 \theta_{2}}{b}>\frac{6 e(1)}{b}
$$

we obtain

$$
\gamma b>6(1-\gamma) e(1) / 4>e(1)
$$

Proposition 3.5. Assume $b \geq 6$. If $k_{i+1}-k_{i}= \pm 1 \bmod b$ for each $i \in$ $\{1,2, \ldots, e(1)-1\}$ then $e(1)<\gamma b$.

Proof. For definiteness of notation, let us assume $k_{2}-k_{1}=1 \bmod b$. Then since $k_{1}, k_{2}, \cdots, k_{e(1)} \in\{0,1, \ldots, b-1\}$, we have $k_{i+1}-k_{i}=1 \bmod b$ for each $1 \leq i<e(1)$. Put $y^{i}=\pi\left(2 k_{1}+2 i-1+2 z\right) / b$. Then for each $1 \leq i<e(1)$,

$$
\cos \left(2 \pi x_{i+1}\right)-\cos \left(2 \pi x_{i}\right)=-2 \sin \frac{\pi}{b} \sin y^{i},
$$

and

$$
0 \leq \sin \left(2 \pi x_{i+1}\right)-\sin \left(2 \pi x_{i}\right)=2 \sin \frac{\pi}{b} \cos y^{i} .
$$

By (3.13), it follows that

$$
\left|\sin y^{i}\right| \leq \frac{2 \gamma}{(b-\gamma) \sin (\pi / b)}<0.8 \gamma,
$$

where we use

$$
(b-\gamma) \sin (\pi / b)>(b-1) \sin (\pi / b) \geq 5 \sin (\pi / 6)=5 / 2 .
$$

Therefore,

$$
y^{i} \in \bigcup_{n \in \mathbb{Z}}(n \pi-\arcsin (0.8 \gamma), n \pi+\arcsin (0.8 \gamma)) .
$$


For each $1 \leq i<e(1)-1, y^{i}$ and $y^{i+1}$ must lie in the same component of the last set, since

$$
y^{i+1}-y^{i}=\frac{2 \pi}{b} \leq \frac{\pi}{3}<\pi-2 \arcsin (0.8 \gamma) .
$$

Therefore, there exists $n_{0} \in \mathbb{Z}$ such that

$$
y^{i}-n_{0} \pi \in(-\arcsin (0.8 \gamma), \arcsin (0.8 \gamma)) \text { for each } i \in\{1,2, \ldots, e(1)-1\} .
$$

Consequently,

$$
e(1)-2=\frac{y^{e(1)-1}-y^{1}}{2 \pi / b}<\frac{2 \arcsin (0.8 \gamma)}{2 \pi / b} \leq 0.4 \gamma b,
$$

where we used $\arcsin t \leq \pi t / 2$ for each $t \in[0,1]$. If $2+0.4 \gamma b \leq \gamma b$, then we are done. So assume the contrary. Then $\gamma b<10 / 3$ and hence $e(1)-2<4 / 3$. Therefore $e(1) \leq 3$. If $\gamma>1 / 2$, then $e(1)<\gamma b$ holds. So assume $\gamma \leq 1 / 2$. Then

$$
\frac{\arcsin (0.8 \gamma)}{0.8 \gamma} \leq \frac{\arcsin (0.5)}{0.5}=\frac{\pi}{3}
$$

and hence (3.22) improves to the following $e(1)-2<4 \gamma b / 15$. If $2+4 \gamma b / 15 \leq \gamma b$ then we are done. So assume $2+4 \gamma b / 15>\gamma b$. Then $\gamma b<30 / 11$ and hence $e(1)-2<4 \gamma b / 15<1$. It follows that $e(1)=1$ or 2 . If $\gamma b>2$ then $e(1)<\gamma b$. So assume $\gamma b \leq 2$. To complete the proof we need to show $e(1)=1$. By (3.5), it suffices to show

$$
\left(\frac{2 \gamma}{b-\gamma}\right)^{2}+\left(\frac{2 \gamma \Delta_{b, \gamma}}{1-\gamma^{2}}\right)^{2}<4 \sin ^{2} \frac{\pi}{b}
$$

Since $\gamma b \leq 2$, we are reduced to show

$$
\frac{16}{\left(b^{2}-2\right)^{2}}+\frac{16}{(b-2)^{2}}\left(\frac{\Delta_{b, 2 / b}}{1+2 / b}\right)^{2}<4 \sin ^{2} \frac{\pi}{b} \text {. }
$$

In the case $b=6$, by (3.1), $\Delta_{6,1 / 3} \leq \max (0.99+1 / 3,1+0.972 / 3)=1.324$, then an easy numerical calculation shows that the left hand side of (3.23) is less than the right hand side which is equal to 1 . Assume now $b \geq 7$. Using $\Delta_{b, 2 / b} \leq 1+2 / b$, we are further reduced to show

$$
\frac{4 b^{2}}{\left(b^{2}-2\right)^{2}}+\frac{4 b^{2}}{(b-2)^{2}}<b^{2} \sin ^{2} \frac{\pi}{b} \text {. }
$$

Note that the left hand side is decreasing in $b$ and the right hand side is increasing in $b$. Thus it suffices to verify this inequality in the case $b=7$, which is an easy exercise.

3.2. The case $b=5$. We use $\sin (\pi / 5)=\sqrt{10-2 \sqrt{5}} / 4$. By (3.6), for each $1 \leq i<e(1)$, since $\gamma<1$,

$$
\left|\sin \left(2 \pi x_{i}\right)-\sin \left(2 \pi x_{i+1}\right)\right| \geq \sqrt{4 \sin ^{2} \frac{\pi}{5}-(4 / 4)^{2}}=(\sqrt{5}-1) / 2>0.6 .
$$

Moreover, by (3.8) if either $k_{i}=k$ or $k_{i+1}=k$, then

$$
\left|\sin \left(2 \pi x_{i}\right)-\sin \left(2 \pi x_{i+1}\right)\right| \geq \sqrt{4 \sin ^{2} \frac{\pi}{5}-(2 / 4)^{2}}=\sqrt{9-\sqrt{5}} / 2>1 .
$$

Thus

$$
2 \geq\left|\sin \left(2 \pi x_{e(1)}\right)-\sin \left(2 \pi x_{1}\right)\right|>1+0.6(e(1)-2),
$$


which implies $e(1) \leq 3$, since $e(1)$ is an integer. If $\gamma>3 / 5$ then $e(1)<\gamma b$. Assume now $\gamma \leq 3 / 5$. Then by (3.6), for each $1 \leq i<e(1)$,

$$
\left|\sin \left(2 \pi x_{i}\right)-\sin \left(2 \pi x_{i+1}\right)\right| \geq \sqrt{4 \sin ^{2} \frac{\pi}{5}-(3 / 5)^{2}}=\sqrt{(4.64-\sqrt{5}) / 2}>1 .
$$

Thus $2 \geq 1.1+(e(1)-2)$ which implies $e(1) \leq 2$.

3.3. The case $b=4$. We use $\sin (\pi / 4)=\sqrt{2} / 2$. By (3.6), for each $1 \leq i<e(1)$, since $\gamma<1$,

$$
\left|\sin \left(2 \pi x_{i}\right)-\sin \left(2 \pi x_{i+1}\right)\right| \geq \sqrt{4 \sin ^{2} \frac{\pi}{4}-(4 / 3)^{2}}=\frac{\sqrt{2}}{3} .
$$

Moreover, by (3.8), if $k_{i}=k$ or $k_{i+1}=k$, then

$$
\left|\sin \left(2 \pi x_{i}\right)-\sin \left(2 \pi x_{i+1}\right)\right| \geq \sqrt{4 \sin ^{2} \frac{\pi}{4}-(2 / 3)^{2}}=\frac{\sqrt{14}}{3} .
$$

Thus

$$
2 \geq\left|\sin \left(2 \pi x_{e(1)}\right)-\sin \left(2 \pi x_{1}\right)\right| \geq \frac{\sqrt{14}}{3}+(e(1)-2) \frac{\sqrt{2}}{3},
$$

which implies $e(1) \leq 3$. Therefore, either $e(1)<\gamma b$ or $\gamma \leq 3 / 4$. Assume the latter. Then by (3.6), for each $1 \leq i<e(1)$ we have

$$
\left|\sin \left(2 \pi x_{i}\right)-\sin \left(2 \pi x_{i+1}\right)\right| \geq \sqrt{4 \sin ^{2} \frac{\pi}{4}-1}=1 .
$$

Thus $2 \geq \sqrt{14} / 3+(e(1)-2)$, which implies $e(1) \leq 2$.

3.4. The case $b=3$. We use $\sin (\pi / 3)=\sqrt{3} / 2$. We claim that for each $z \in[0,1], E(1, z) \neq\{0,1,2\}^{2}$, so that by Lemma 2.7, $\sigma(1) \leq \sqrt{2}+1$. Otherwise, there exists $z \in[0,1]$ such that $E(1, z)=\{0,1,2\}^{2}$. Using the notation introducded above, for any $1 \leq i<j \leq 3$, as in (3.8), we have

$$
\left|\sin \left(2 \pi x_{i}\right)-\sin \left(2 \pi x_{j}\right)\right| \geq \sqrt{4 \sin ^{2} \frac{\pi}{3}-\frac{4}{4}}=\sqrt{2},
$$

which contradictis the fact

$2 \geq \sin \left(2 \pi x_{3}\right)-\sin \left(2 \pi x_{1}\right)=\left|\sin \left(2 \pi x_{3}\right)-\sin \left(2 \pi x_{2}\right)\right|+\left|\sin \left(2 \pi x_{2}\right)-\sin \left(2 \pi x_{1}\right)\right|$.

Assume $\sigma(1) \geq \gamma b$. Then $\gamma<(1+\sqrt{2}) / 3<0.81$. Keep the notation $x_{j}$, $e(1)$ as above. By (3.6), for each $1 \leq i<e(1)$ we have

$$
\left|\sin \left(2 \pi x_{i}\right)-\sin \left(2 \pi x_{i+1}\right)\right| \geq \sqrt{4 \sin ^{2} \frac{\pi}{3}-\frac{16 \gamma^{2}}{(3-\gamma)^{2}}}>0.9 .
$$

By (3.8), if $k_{i}=k$ or $k_{i+1}=k$, then $\left|\sin \left(2 \pi x_{i}\right)-\sin \left(2 \pi x_{i+1}\right)\right| \geq \sqrt{2}$. Thus $2 \geq \sqrt{2}+(e(1)-2) \cdot 0.9$ which implies that $e(1) \leq 2$. 


\section{Proof of Theorem 2.3. The case $b=3,4,5$}

In this section, we shall prove Theorem 2.3 in the case $b \in\{3,4,5\}$. We shall need the following improvment of Lemma 3.2.

Lemma 4.1. Let $x^{*} \in[0,1 / 2]$ and $0 \leq k<l<b$ be such that $(k, l) \in E\left(1, x^{*}\right)$. Then for any $\kappa \in(0,1)$, one of the following holds: either

$$
\left|\cos \frac{2 \pi\left(x^{*}+k\right)}{b}-\cos \frac{2 \pi\left(x^{*}+l\right)}{b}\right| \leq \frac{2 \gamma \sqrt{1-\kappa^{2}}}{b}+\frac{2 \gamma^{2}}{b(b-\gamma)},
$$

or

$$
\left|\sin \frac{2 \pi\left(x^{*}+k\right)}{b}-\sin \frac{2 \pi\left(x^{*}+l\right)}{b}\right| \leq 2 \kappa \gamma+\frac{2 \gamma^{2}}{1-\gamma}
$$

Proof. By Proposition 2.2 (1), there exist $\mathbf{k}=\left\{k_{n}\right\}_{n=1}^{\infty}$ and $\mathbf{l}=\left\{l_{n}\right\}_{n=1}^{\infty}$ in $\mathcal{A}^{\mathbb{Z}^{+}}$ with $k_{1}=k$ and $l_{1}=l$ and such that the function

$$
F(x)=-\frac{1}{2 \pi}(S(x, \mathbf{k})-S(x, \mathbf{l})),
$$

has a multiple zero at $x=x^{*}$. Let

$$
x_{n}=\frac{x+k_{1}+b k_{2}+\cdots+b^{n-1} k_{n}}{b^{n}}, y_{n}=\frac{x+l_{1}+b l_{2}+\cdots+b^{n-1} l_{n}}{b^{n}},
$$

and let

$$
P_{n}(x)=\sin \left(2 \pi x_{n}\right)-\sin \left(2 \pi y_{n}\right), Q_{n}(x)=\cos \left(2 \pi x_{n}\right)-\cos \left(2 \pi y_{n}\right) .
$$

Since $F\left(x^{*}\right)=\sum_{n=1}^{\infty} \gamma^{n-1} P_{n}\left(x^{*}\right)$, we have

$$
\left|P_{1}\left(x^{*}\right)\right| \leq \gamma\left|P_{2}\left(x^{*}\right)\right|+\sum_{n=3}^{\infty} 2 \gamma^{n-1}=\gamma\left|P_{2}\left(x^{*}\right)\right|+\frac{2 \gamma^{2}}{1-\gamma} .
$$

If $\left|P_{2}\left(x^{*}\right)\right| \leq 2 \kappa$, then this implies that (4.2) holds. Assume $\left|P_{2}\left(x^{*}\right)\right|>2 \kappa$. Since $P_{2}\left(x^{*}\right)^{2}+Q_{2}\left(x^{*}\right)^{2} \leq 4$, we have $\left|Q_{2}\left(x^{*}\right)\right| \leq 2 \sqrt{1-\kappa^{2}}$. Since $0=\frac{b F^{\prime}\left(x^{*}\right)}{2 \pi}=$ $\sum_{n=1}^{\infty}(\gamma / b)^{n-1} Q_{n}\left(x^{*}\right)$, we conclude

$$
\left|Q_{1}\left(x^{*}\right)\right| \leq \frac{\gamma}{b}\left|Q_{2}\left(x^{*}\right)\right|+\sum_{n=3}^{\infty} 2\left(\frac{\gamma}{b}\right)^{n-1} \leq \frac{2 \gamma \sqrt{1-\kappa^{2}}}{b}+\frac{2 \gamma^{2}}{b(b-\gamma)},
$$

which is (4.1).

4.1. The case $b=5$. By Theorem 3.1 (ii), to complete the proof of Theorem 2.3 in the case $b=5$, it suffices to prove the following.

Theorem 4.1. Assume $b=5$ and $e(1)=2$. Then $\sigma(1)<5 \gamma$.

Lemma 4.2. Assume $\gamma \leq 2 / 5$. Let $0 \leq x^{*} \leq 1 / 2$ and $0 \leq k<l<5$ be such that $(k, l) \in E\left(1, x^{*}\right)$. Then either

$$
0 \leq x^{*}<1 / 10 \text { and }(k, l)=(2,3),
$$

or

$$
2 / 5<x^{*} \leq 1 / 2 \text { and }(k, l)=(0,4)
$$


Proof. Put $x^{*}(k)=\left(x^{*}+k\right) / 5, x^{*}(l)=\left(x^{*}+l\right) / 5$, and $y^{*}=\pi\left(2 x^{*}+k+l\right) / 5$. We shall use Lemma 3.2 and Lemma 4.1 to prove

$$
\left|\sin y^{*}\right|<\sin \frac{\pi}{25}
$$

which implies the statement.

By (3.4) and (3.5) in Lemma 3.2, we have

$$
\left|\cos \left(2 \pi x^{*}(k)\right)-\cos \left(2 \pi x^{*}(l)\right)\right| \leq \frac{2 \gamma}{5-\gamma}=\frac{4}{23},
$$

and

$$
4 \sin ^{2} \frac{\pi(l-k)}{5} \leq\left(\frac{2 \gamma}{1-\gamma}\right)^{2}+\left(\frac{2 \gamma}{5-\gamma}\right)^{2}<\left(\frac{4}{3}\right)^{2}+\left(\frac{4}{23}\right)^{2}<2 .
$$

The latter inequality implies that $l-k= \pm 1 \bmod 5$.

Let $\kappa=\sqrt{2} / 2$. Let us show that the inequality (4.2) does not hold. Indeed, otherwise, we would have

$$
\left|\sin \left(2 \pi x^{*}(k)\right)-\sin \left(2 \pi x^{*}(l)\right)\right| \leq \gamma \sqrt{2}+\frac{2 \gamma^{2}}{1-\gamma}<1.1,
$$

which together with (4.6) would imply that

$$
\begin{aligned}
& 1.38 \cdots=4 \sin ^{2} \frac{\pi}{5}=4 \sin ^{2} \frac{\pi(l-k)}{5} \\
& =\left|\cos \left(2 \pi x^{*}(k)\right)-\cos \left(2 \pi x^{*}(l)\right)\right|^{2}+\left|\sin \left(2 \pi x^{*}(k)\right)-\sin \left(2 \pi x^{*}(l)\right)\right|^{2} \\
& <(4 / 23)^{2}+1.1^{2}<1.3,
\end{aligned}
$$

which is absurd.

Therefore the inequality (4.1) holds. It follows that

$$
2 \sin \frac{\pi}{5}\left|\sin y^{*}\right|=\left|\cos \left(2 \pi x^{*}(k)\right)-\cos \left(2 \pi x^{*}(l)\right)\right| \leq \frac{\gamma \sqrt{2}}{5}+\frac{2 \gamma^{2}}{5(5-\gamma)}<0.128,
$$

and hence $\left|\sin \left(y^{*}\right)\right|<0.11<\sin (\pi / 25)$.

Lemma 4.3. If $\gamma \leq(\sqrt{5}+1) / 10$, then $e(1)=1$.

Proof. Suppose $(k, l) \in E(1, x)$. Then by (3.5) in Lemma 3.2, we obtain

$$
4 \sin ^{2} \frac{(l-k) \pi}{5}<\left(\frac{2 \gamma}{5-\gamma}\right)^{2}+\left(\frac{2 \gamma}{1-\gamma}\right)^{2}<4 \sin ^{2} \frac{\pi}{5}
$$

which implies that $k=l$.

Proof of Theorem 4.1. If $\gamma>2 / 5$, then $\sigma(1) \leq e(1)=2<5 \gamma$. Assume now $\gamma \leq 2 / 5$ so that Lemma 4.2 applies. By Proposition 2.2 (3), there exist $\varepsilon>0$ and $\delta>0$ such that if $(k, l) \in E(1, x ; \varepsilon, \delta)$ for some $x \in[0,1 / 2]$, then we have either $x \in[0,1 / 10),(k, l)=(2,3)$ or $x \in(2 / 5,1 / 2],(k, l)=(0,4)$.

Let $K=[1 / 10,2 / 5] \cup[3 / 5,9 / 10]$. Then by Lemma 2.9 $e(1, x ; \varepsilon, \delta)=1$ for all $x \in K$ and $e(1, x ; \varepsilon, \delta) \leq 2$ for all $x \in[0,1)$, so the condition (i) in Lemma 2.11 is satisfied (for $q=1$ ). Let us prove that the condition (ii) is satisfied. Let $x \in[0,1) \backslash K$ and $0 \leq k<l<5$ be such that $(k, l) \in E(1, x ; \varepsilon, \delta)$. We need to check either $x(k) \in K$ or $x(l) \in K$. Indeed, by symmetry (Lemma 2.9), it suffices to consider the case $x \in[0,1 / 2] \backslash K$; while for $x \in[0,1 / 10)$, we have $(k, l)=(2,3)$ and $x(3) \in K$ and for $x \in(2 / 5,1 / 2]$ we have $(k, l)=(0,4)$ 
and $x(4) \in K$. Having verified the conditions in Lemma 2.11, we conclude $\sigma(1) \leq(\sqrt{5}+1) / 2$. By Lemma 4.3, $\gamma>(\sqrt{5}+1) / 10$ since we assume $e(1)=2$. Thus $\sigma(1)<5 \gamma$.

4.2. The case $b=4$. By Theorem 3.1 (ii), to complete the proof of Theorem 2.3 in the case $b=4$, it suffices to prove the following.

Theorem 4.2. Assume $b=4$ and $e(1)=2$. Then $\sigma(1)<b \gamma$.

First we apply Lemma 3.2 and Lemma 4.1 to obtain the following estimate.

Lemma 4.4. Assume $\gamma \leq 1 / 2$. Let $0 \leq x^{*} \leq 1 / 2$ and let $0 \leq k<l<4$ be such that $(k, l) \in E\left(1, x^{*}\right)$. Then either

$$
x^{*} \in[3 / 8,1 / 2] \text { and }(k, l) \in\{(0,3),(1,2)\},
$$

or

$$
x^{*} \in[0,1 / 8] \text { and }(k, l)=(1,3) .
$$

Proof. By Lemma 3.2 ,

$$
\left|\cos \frac{2 \pi\left(x^{*}+k\right)}{4}-\cos \frac{2 \pi\left(x^{*}+l\right)}{4}\right| \leq \frac{2 \gamma}{4-\gamma} \leq \frac{2}{7} .
$$

Let us apply Lemma 4.1 with $\kappa=1 / 3$. We claim that (4.2) does not hold. Indeed, otherwise,

$$
\left|\sin \frac{2 \pi\left(x^{*}+k\right)}{4}-\sin \frac{2 \pi\left(x^{*}+l\right)}{4}\right| \leq \frac{4}{3},
$$

which together with (4.7) would imply that

$$
\begin{aligned}
& 2 \leq 4 \sin ^{2} \frac{\pi(l-k)}{4} \\
&=\left(\cos \frac{2 \pi\left(x^{*}+k\right)}{4}-\cos \frac{2 \pi\left(x^{*}+l\right)}{4}\right)^{2}+\left(\sin \frac{2 \pi\left(x^{*}+k\right)}{4}-\sin \frac{2 \pi\left(x^{*}+l\right)}{4}\right)^{2} \\
& \leq\left(\frac{2}{7}\right)^{2}+\left(\frac{4}{3}\right)^{2}<2,
\end{aligned}
$$

which is absurd. Therefore, the inequality (4.1) holds with $\kappa=1 / 3$, which implies that

$$
2\left|\sin \frac{\pi(l-k)}{4}\right|\left|\sin \left(\frac{2 \pi x^{*}}{4}+\frac{\pi(k+l)}{4}\right)\right| \leq \frac{\sqrt{2}}{6}+\frac{1}{28} .
$$

Consequently,

$$
\left|\sin \left(\frac{2 \pi x^{*}}{4}+\frac{\pi(k+l)}{4}\right)\right| \leq \frac{1}{6}+\frac{1}{28 \sqrt{2}}<\sin \frac{\pi}{16} .
$$

Since $2 \pi x^{*} / 4 \in[0, \pi / 4]$ the lemma follows.

A bit more careful analysis shows that the seocnd alternative in the lemma above never happens.

Lemma 4.5. Assume $\gamma \leq 1 / 2$. Then for any $x^{*} \in[0,1 / 2],(1,3) \notin E\left(1, x^{*}\right)$. 
Proof. We shall prove this lemma by contradiction. Assume $(1,3) \in E\left(1, x^{*}\right)$. Then there exists $\mathbf{k}=\left\{k_{n}\right\}_{n=1}^{\infty}$ and $\mathbf{l}=\left\{l_{n}\right\}_{n=1}^{\infty}$ with $k_{1}=1$ and $l_{1}=3$ and such that the function

$$
F(x)=-\frac{1}{2 \pi}(S(x, \mathbf{k})-S(x, \mathbf{l}))
$$

has a multiple zero at $x^{*}$. Let

$$
x_{n}=\frac{x+k_{1}+4 k_{2}+\cdots+4^{n-1} k_{n}}{4^{n}}, y_{n}=\frac{x+l_{1}+4 l_{2}+\cdots+4^{n-1} l_{n}}{4^{n}},
$$

and let

$$
P_{n}(x)=\sin \left(2 \pi x_{n}\right)-\sin \left(2 \pi y_{n}\right), Q_{n}(x)=\cos \left(2 \pi x_{n}\right)-\cos \left(2 \pi y_{n}\right) .
$$

Then $F(x)=\sum_{n=1}^{\infty} \gamma^{n-1} P_{n}(x)$. Since $F\left(x^{*}\right)=0$, this gives us

$$
\left|P_{1}\left(x^{*}\right)+\gamma P_{2}\left(x^{*}\right)\right| \leq \sum_{n=3}^{\infty} \gamma^{n-1}\left|P_{n}\left(x^{*}\right)\right| \leq 1 .
$$

Note that

$$
P_{2}(x) \geq-\cos \frac{\pi(1+x)}{4}-\cos \frac{\pi(1-x)}{4}=-2 \cos \frac{\pi}{4} \cos \frac{\pi x}{4} \geq-\sqrt{2} .
$$

Therefore

$$
P_{1}\left(x^{*}\right) \leq 1-\gamma P_{2}\left(x^{*}\right) \leq 1+\frac{\sqrt{2}}{2} .
$$

As in the previous lemma, $\left|Q_{1}\left(x^{*}\right)\right| \leq 2 / 7$. Since $P_{1}\left(x^{*}\right)>0$, we have

$$
P_{1}\left(x^{*}\right)^{2}+Q_{1}\left(x^{*}\right)^{2} \leq(2 / 7)^{2}+(1+\sqrt{2} / 2)^{2}<4 .
$$

However, the left hand of the inequality is equal to 4 , a contradiction!

Lemma 4.6. If $\gamma \leq(\sqrt{5}+1) / 8$ then $e(1)=1$.

Proof. For $x \in[0,1]$ and $0 \leq k<l<4$, if $(k, l) \in E(1, x)$, then by the inequality (3.5) in Lemma 3.2, we have

$$
4 \sin ^{2} \frac{\pi(l-k)}{4}<\left(\frac{2 \gamma}{4-\gamma}\right)^{2}+\left(\frac{2 \gamma}{1-\gamma}\right)^{2}<2,
$$

which implies that $l=k$.

Proof of Theorem 4.2. If $\gamma>1 / 2$, then $\sigma(1) \leq e(1)=2<4 \gamma$. So assume $\gamma \leq$ $1 / 2$. By Lemmas 4.4 and 4.5 and Proposition 2.2 (3), there exist $\varepsilon>0$ and $\delta>0$ such that if $x \in[0,1 / 2]$ and $0 \leq k<l<4$ are such that $(k, l) \in E(1, x ; \varepsilon, \delta)$ then $3 / 8<x \leq 1 / 2$ and $(k, l) \in\{(0,3),(1,2)\}$. Note that for $x \in(3 / 8,1 / 2]$, we have $x(0), x(1) \in[0,3 / 8]$. By Lemma [2.9, it is then easy to check that the conditions of Lemma 2.11 are satisfied for $K=[0,3 / 8] \cup[5 / 8,1)$ and $q=1$. Thus $\sigma(1) \leq(\sqrt{5}+1) / 2$. On the other hand, since we assume $e(1)=2$, by Lemma 4.6. we have $\gamma>(\sqrt{5}+1) / 8$. This proves that $\sigma(1)<4 \gamma$. 
4.3. The case $b=3$. In this subsection, we shall prove the following theorem, which together with Theorem 3.1, implies Theorem 2.3 in the case $b=3$.

Theorem 4.3. Assume $b=3$ and $e(1)=2$. Then $\sigma(1)<b \gamma$.

Lemma 4.7. Assume $\gamma \leq 2 / 3$. Let $0 \leq x^{*} \leq 1 / 2$ and let $0 \leq k<l<3$ be such that $(k, l) \in E(1, x)$. Then either

$$
x \in[0,1 / 6) \text { and }(i, j)=(1,2) ;
$$

or

$$
x \in(1 / 3,1 / 2] \text { and }(i, j)=(0,2) .
$$

Proof. If $(k, l) \in E\left(1, x^{*}\right)$, then by (3.4),

$$
\left|\cos \frac{2 \pi\left(x^{*}+k\right)}{3}-\cos \frac{2 \pi\left(x^{*}+l\right)}{3}\right| \leq \frac{2 \gamma}{3-\gamma} \leq \frac{4}{7},
$$

which implies that

$$
\left|\sin \left(\frac{2 \pi x^{*}}{3}+\frac{\pi(k+l)}{3}\right)\right| \leq \frac{4}{7 \cdot 2\left|\sin \frac{\pi(l-k)}{3}\right|}=\frac{4}{7 \sqrt{3}}<\sin \frac{\pi}{9} .
$$

The statement follows.

Lemma 4.8. Assume $\gamma \leq(\sqrt{5}+1) / 6$. Let $0 \leq x \leq 1 / 2$ and let $0 \leq k<l<3$ be such that $(k, l) \in E(1, x)$. Then either

$$
x \in[0,1 / 8] \text { and }(k, l)=(1,2) ;
$$

or

$$
x \in[3 / 8,1 / 2] \text { and }(k, l)=(0,2) .
$$

Proof. Under current assumption, again by (3.4), we have

$$
\left|\cos \frac{2 \pi\left(x^{*}+k\right)}{3}-\cos \frac{2 \pi\left(x^{*}+l\right)}{3}\right| \leq \frac{2 \gamma}{3-\gamma} \leq 0.44,
$$

hence

$$
\left|\sin \left(\frac{2 \pi x^{*}}{3}+\frac{\pi(k+l)}{3}\right)\right| \leq \frac{0.44}{2\left|\sin \frac{\pi(l-k)}{3}\right|}=\frac{0.44}{\sqrt{3}}<\sin \frac{\pi}{12} .
$$

Thus the statement holds.

Lemma 4.9. If $3 \gamma \leq \sqrt{2}$, then $e(1)=1$.

Proof. By (3.2) and (3.5), if $(k, l) \in E\left(1, x^{*}\right)$ for some $0 \leq k, l<3$ and $x^{*} \in \mathbb{R}$, then

$$
4 \sin ^{2} \frac{\pi(l-k)}{3} \leq\left(\frac{2 \gamma}{3-\gamma}\right)^{2}+\left(\frac{2 \gamma(1+0.71 \gamma)}{1-\gamma^{2}}\right)^{2} .
$$

Since $\gamma \leq \sqrt{2} / 3$, the right hand side is less than 3 , which implies that $k=l$. This proves that $e(1)=1$.

Proof of Theorem 4.3. If $\gamma>2 / 3$, then $\sigma(1) \leq e(1)=2<3 \gamma$. So assume $\gamma \leq 2 / 3$. By Lemma 4.7 and Proposition 2.2 (3), there exist $\varepsilon>0$ and $\delta>0$ such that if $x \in[0,1 / 2], 0 \leq k<l<3$ are such that $(k, l) \in E(1, x ; \varepsilon, \delta)$, then either $x \in[0,1 / 6),(k, l)=(1,2)$ or $x \in(1 / 3,1 / 2],(k, l)=(0,2)$. Note that for $x \in[0,1 / 6)$, we have $x(2) \in[2 / 3,5 / 6]$ and for $x \in(1 / 3,1 / 2]$, we have 
$x(0) \in[1 / 6,1 / 3]$. By Lemma 2.9 it follows that the conditions in Lemma 2.11 are satisfied with $K=[1 / 6,1 / 3] \cup[2 / 3,5 / 6]$ and $q=1$. Thus $\sigma(1) \leq(\sqrt{5}+1) / 2$.

If $\gamma>(\sqrt{5}+1) / 6$, then $\sigma(1)<3 \gamma$. Assume $\gamma \leq(\sqrt{5}+1) / 6$. Then by Lemma 4.8 and Proposition 2.2 (3), there exist $\varepsilon>0$ and $\delta>0$ such that if $x \in[0,1 / 2], 0 \leq k<l<3$ are such that $(k, l) \in E(1, x ; \varepsilon, \delta)$, then either $x \in[0,1 / 8),(k, l)=(1,2)$ or $x \in(3 / 8,1 / 2],(k, l)=(0,2)$. Note that for $x \in[0,1 / 8)$, we have $x(1) \in[1 / 8,3 / 8], x(2) \in[5 / 8,7 / 8]$ and for $x \in[3 / 8,1 / 2]$ we have $x(0) \in[1 / 8,3 / 8]$ and $x(2) \in[5 / 8,7 / 8]$. By Lemma 2.9, it follows that the conditions in Lemma 2.10 are satisfied with $K=[0,3 / 8] \cup[5 / 8,1)$ and $q=1$. Thus $\sigma(1) \leq \sqrt{2}$. Since we assume $e(1)=2$, by Lemma 4.9] $\gamma>\sqrt{2} / 3$. This proves $\sigma(1)<3 \gamma$.

\section{The CASE $b=2$}

This section is devoted to the proof of Theorem 2.3 in the case $b=2$. The proof is structurally similar to the cases $b=3,4,5$ which we discussed above, but it is more involved and consists of several steps.

We shall use the following notation. For any $\mathbf{k}, \mathbf{l} \in \mathcal{A}^{q}$ and $x_{*} \in \mathbb{R}$, we write $\mathbf{k} \sim_{x_{*}} \mathbf{l}$ if $(\mathbf{k}, \mathbf{l}) \in E\left(q, x_{*}\right)$. In order to show $\mathbf{k} \varkappa_{x_{*}} \mathbf{l}$, by Proposition 2.2 (1), it suffices to show that any $\mathbf{u}, \mathbf{v} \in \mathcal{A}^{\mathbb{Z}^{+}}$, the function $S(x, \mathbf{k u})-S(x, \mathbf{l v})$ does not have a multiple zero at $x_{*}$.

5.1. Step 1. When $\gamma>\sqrt{2} / 2$. In this step, we shall prove

Proposition 5.1. (1) For any $\gamma \in(1 / 2,1), \sigma(1) \leq(\sqrt{5}+1) / 2$.

(2) For any $\gamma \in(1 / 2,(\sqrt{5}+1) / 4], \sigma(1) \leq \sqrt{2}$.

An immediate corollary is the following:

Corollary 5.2. Assume $b=2$ and $\gamma>\sqrt{2} / 2$. Then $\sigma(1)<2 \gamma$.

The proof of this proposition relies on the following estimates.

Lemma 5.3. (i) For any $x \in[0,1 / 3]$, we have $(00) \varkappa_{x}(10),(00) \varkappa_{x}(11)$, and $(01) \varkappa_{x}(10)$.

(ii) If either $x \in[0,1 / 4]$, or $x \in[0,1 / 3]$ and $\gamma \leq(\sqrt{5}+1) / 4$, then $(01) \chi_{x}$ (11).

Proof. Let $\mathbf{u}=\left\{u_{n}\right\}_{n=1}^{\infty}$ and $\mathbf{v}=\left\{v_{n}\right\}_{n=1}^{\infty}$ be elements in $\mathcal{A}^{\mathbb{Z}^{+}}$and let

$$
F(x)=-\frac{1}{2 \pi}(S(x, \mathbf{u})-S(x, \mathbf{v})) .
$$

As before, given $x \in \mathbb{R}$, we write

$$
x_{n}=\frac{x}{2^{n}}+\frac{u_{1}}{2^{n}}+\cdots+\frac{u_{n}}{2}, y_{n}=\frac{x}{2^{n}}+\frac{v_{1}}{2^{n}}+\cdots+\frac{v_{n}}{2}
$$

and write $Q_{n}=\cos \left(2 \pi x_{n}\right)-\cos \left(2 \pi y_{n}\right)$. Then $\left|Q_{n}\right| \leq 2$ for all $n$ and

$$
G(x):=\frac{F^{\prime}(x)}{\pi}=\sum_{n=1}^{\infty}\left(\frac{\gamma}{2}\right)^{n-1} Q_{n} .
$$

In the following, we assume $u_{1}=0$ and $v_{1}=1$, so that $Q_{1}=2 \cos (\pi x)$. 
(i). Assume first $u_{2}=0$. Then

$$
Q_{2}=\cos \frac{\pi x}{2}+(-1)^{v_{2}} \sin \frac{\pi x}{2} \geq \cos \frac{\pi x}{2}-\sin \frac{\pi x}{2}>0
$$

for any $x \in[0,1 / 2]$. Thus

$$
G(x) \geq Q_{1}-\sum_{n=3}^{\infty}(\gamma / 2)^{n-1}\left|Q_{n}\right|>2 \cos (\pi x)-1 \geq 0 .
$$

whenever $0 \leq x \leq 1 / 3$. This proves that for any $x \in[0,1 / 3],(00) \chi_{x}(10)$ and $(00) \chi_{x}(11)$

To prove $(01) \chi_{x}(10)$ for $x \in[0,1 / 3]$, let $u_{2}=1$ and $v_{2}=0$. Then

$$
Q_{2}=\sin (\pi x / 2)-\cos (\pi x / 2),
$$

and

$$
Q_{3}= \pm \sin (\pi x / 4) \pm \cos (\pi(1+x) / 4) \geq-\sin (\pi / 12)-\cos (\pi / 4)>-1 .
$$

Thus

$$
\begin{aligned}
G(x) & \geq Q_{1}+\frac{\gamma}{2} Q_{2}+\frac{\gamma^{2}}{4} Q_{3}+\sum_{n=3}^{\infty} \frac{\gamma^{n}}{2^{n}} Q_{n+1} \\
& \geq g(x)-\frac{\gamma^{2}}{4}-\frac{\gamma^{3}}{4-2 \gamma} \geq g(x)-\frac{3}{4}
\end{aligned}
$$

where

Since

$$
g(x)=2 \cos (\pi x)+\frac{1}{2}\left(\sin \frac{\pi x}{2}-\cos \frac{\pi x}{2}\right) .
$$

$$
g^{\prime \prime}(x)=-2 \pi^{2} \cos (\pi x)-\pi^{2} / 8(\sin (\pi x / 2)-\cos (\pi x / 2)<0
$$

holds for all $x \in[0,1 / 3]$, we have

$$
\min _{x \in[0,1 / 3]} g(x)=\min (g(0), g(1 / 3))=\min \left(\frac{3}{2}, \frac{5-\sqrt{3}}{4}\right)>\frac{3}{4} .
$$

Therefore $G>0$.

(ii) Now let us assume $u_{2}=v_{2}=1$. Then

$$
Q_{2}=-\sin \frac{\pi x}{2}-\cos \frac{\pi x}{2}
$$

and

$$
Q_{3}= \pm \sin \frac{\pi x}{4} \pm \sin \frac{\pi(1+x)}{4} \geq-2 \sin \frac{\pi(1+2 x)}{8} \cos \frac{\pi}{8}
$$

Thus

$$
G(x) \geq Q_{1}+\frac{\gamma}{2} Q_{2}+\frac{\gamma^{2}}{4} Q_{3}-2 \sum_{n=4}^{\infty}\left(\frac{\gamma}{2}\right)^{n-1} \geq h_{\gamma}(x)-\frac{\gamma^{3}}{4-2 \gamma},
$$

where

$$
h_{\gamma}(x)=2 \cos (\pi x)-\frac{\gamma}{2}\left(\cos \frac{\pi x}{2}+\sin \frac{\pi x}{2}\right)-\frac{\gamma^{2}}{2} \cos \frac{\pi}{8} \sin \frac{\pi(1+2 x)}{8} .
$$

Assume first $x \in[0,1 / 4]$. Since $h_{\gamma}(x)$ is decreasing in both $x$ and $\gamma$, we have

$$
h_{\gamma}(x) \geq h_{1}(1 / 4)=\sqrt{2}-\frac{\sqrt{2}}{2} \sin \frac{3 \pi}{8}-\frac{1}{2} \cos \frac{\pi}{8} \sin \frac{3 \pi}{16}>\frac{1}{2}>\frac{\gamma^{3}}{4-2 \gamma},
$$


hence $G(x)>0$. This proves that $(01) \varkappa_{x}(11)$ for $x \in[0,1 / 4]$.

Assume now $\gamma \leq(\sqrt{5}+1) / 4=: \gamma_{0}$. Then by numerical calculation, we have

$$
h_{\gamma_{0}}(1 / 3)=1-\frac{\gamma_{0}}{2} \frac{\sqrt{3}+1}{2}-\frac{\gamma_{0}^{2}}{2} \sin \frac{\pi}{8} \sin \frac{5 \pi}{24}>\frac{\gamma_{0}^{3}}{4-2 \gamma_{0}} .
$$

Thus for each $0 \leq x \leq 1 / 3$, we have

$$
G(x) \geq h_{\gamma}(x)-\gamma^{3} /(4-2 \gamma) \geq h_{\gamma_{0}}(1 / 3)-\gamma_{0}^{3} /\left(4-2 \gamma_{0}\right)>0 .
$$

This proves that $(01) \chi_{x}(11)$ for all $x \in[0,1 / 3]$.

Proof of Proposition 5.1. By Lemma 5.3 and Proposition 2.2 (3), there exist $\varepsilon>0$ and $\delta>0$ such that $e(1, x ; \varepsilon, \delta)=1$ for $x \in[0,1 / 4]$. By Lemma 2.9. this also holds for $x \in[3 / 4,1]$. For $x \in[1 / 4,1 / 2], x(0) \in[0,1 / 4]$ while for $x \in[1 / 2,3 / 4]$ we have $x(1) \in[3 / 4,1]$. By Lemma 2.11, we obtain $\sigma(1) \leq$ $(\sqrt{5}+1) / 2$.

Assume that $\gamma \leq(\sqrt{5}+1) / 4$. Then Lemma 5.3 and Proposition 2.2 (3), there exist $\varepsilon>0$ and $\delta>0$ such that $e(1, x ; \varepsilon, \delta)=1$ for all $x \in[0,1 / 3]$ and by Lemma 2.9. this also holds for $x \in[2 / 3,1]$. For $x \in(1 / 3,2 / 3)$, both $x(0)$ and $x(1)$ belong to $[0,1 / 3] \cup[2 / 3,1]$. By Lemma 2.10 , it follows that $\sigma(1) \leq \sqrt{2}$.

5.2. Step 2: When $\gamma>0.64$. In this section we shall prove the following

Proposition 5.4. Assume $\gamma \leq \sqrt{2} / 2$. Then $\sigma(2) \leq 1.61$.

As an immediate corollary of this proposition and Corollary 5.2, we have

Corollary 5.5. If $\gamma>0.64$ then either $\sigma(1)<2 \gamma$ or $\sigma(2)<(2 \gamma)^{2}$.

The proof of Proposition [5.4 relies on the following estimates.

Lemma 5.6. Assume $\gamma \leq \sqrt{2} / 2$. Then

(i) For any $x \in[0,1 / 2]$, (00) $\nsim_{x}(10)$;

(ii) For any $x \in[0,2 / 5],(01) \chi_{x}(10)$ and $(00) \chi_{x}(11)$;

(iii) For any $x \in[0,1 / 2],(00) \not_{x}(11)$;

(iv) For any $x \in[0,2 / 5],(01) \chi_{x}(11)$;

(v) For any $x \in[0,2 / 5], 0 \nsim_{x} 1$;

(vi) For $x \in[1 / 5,1 / 2],(10) \varkappa_{x}(11)$.

Proof. Let $\mathbf{u}, \mathbf{v} \in \mathcal{A}^{\mathbb{Z}^{+}}$and let $F(x)=-(2 \pi)^{-1}(S(x, \mathbf{u})-S(x, \mathbf{v})), G(x)=$ $F^{\prime}(x) / \pi$. For given $x$, we shall use the notations $x_{n}, y_{n}, Q_{n}$ as in Lemma 5.3 and let $P_{n}=\sin \left(2 \pi x_{n}\right)-\sin \left(2 \pi y_{n}\right)$.

(i) Assume $\left(u_{1}, u_{2}\right)=(0,0)$ and $\left(v_{1}, v_{2}\right)=(1,0)$. Then $Q_{1}=2 \cos (\pi x)$ and $Q_{2}=\cos \frac{\pi x}{2}+\sin \frac{\pi x}{2}$. Thus

$$
G(x)=Q_{1}+\frac{\gamma}{2} Q_{2}+\cdots \geq f(x)-\frac{\gamma^{2}}{2-\gamma},
$$

where

$$
f(x)=2 \cos (\pi x)+\frac{\gamma}{2}\left(\sin \frac{\pi x}{2}+\cos \frac{\pi x}{2}\right) .
$$

On the interval $x \in[0,1 / 2]$, we have

$$
f^{\prime \prime}(x)=-2 \pi^{2} \sin (\pi x)-\frac{\pi^{2} \gamma}{8}\left(\sin \frac{\pi x}{2}+\cos \frac{\pi x}{2}\right)<0 .
$$


Thus

$$
\min _{x \in[0,1 / 2]} f(x)=\min (f(0), f(1 / 2))=\min \left(2+\frac{\gamma}{2}, \frac{\gamma}{\sqrt{2}}\right)=\frac{\gamma}{\sqrt{2}}>\frac{\gamma^{2}}{2-\gamma},
$$

hence $G(x)>0$. Therefore, for any $x \in[0,1 / 2],(00) \chi_{x}(11)$.

(ii) Assume either $\left(u_{1}, u_{2}\right)=(0,1)$ and $\left(v_{1}, v_{2}\right)=(1,0)$; or $\left(u_{1}, u_{2}\right)=(0,0)$ and $\left(v_{1}, v_{2}\right)=(1,1)$. Then

$$
Q_{2}= \pm\left(\cos \frac{\pi x}{2}-\sin \frac{\pi x}{2}\right) \geq \sin \frac{\pi x}{2}-\cos \frac{\pi x}{2} \text {. }
$$

Thus

$$
\begin{aligned}
G(x) & \geq 2 \cos (\pi x)-\frac{\gamma}{2}\left(\cos \frac{\pi x}{2}-\sin \frac{\pi x}{2}\right)-\frac{\gamma^{2}}{2-\gamma} \\
& \geq 2 \cos (\pi x)-\frac{1}{2} \sin \frac{\pi(1-2 x)}{4}-\frac{4+\sqrt{2}}{14}:=g(x)
\end{aligned}
$$

For $x \in[0,2 / 5]$, we have

$$
g^{\prime \prime}(x)=-2 \pi^{2} \cos (\pi x)+\frac{\pi^{2}}{8} \sin \frac{\pi(1-2 x)}{4}<0 .
$$

Thus $g(x) \geq \min (g(0), g(2 / 5))$. Clearly, $g(0)>0$ and $g(2 / 5)>0$. It follows that $G(x)>0$ for all $x \in[0,2 / 5]$. This proves that $(01) \chi_{x}(10)$ and $(00) \chi_{x}$ (11) for $x \in[0,2 / 5]$.

(iii) Assume $\left(u_{1}, u_{2}\right)=(0,0)$ and $\left(v_{1}, v_{2}\right)=(1,1)$. By (ii), it suffices to show that $F(x)>0$ for any $x \in[2 / 5,1 / 2]$. Note that $P_{1}=2 \sin (\pi x)$,

$$
P_{2}=\sin \frac{\pi x}{2}+\cos \frac{\pi x}{2}=\sqrt{2} \sin \left(\frac{\pi x}{2}+\frac{\pi}{4}\right)
$$

and

$$
P_{3} \geq-\sin \frac{\pi x}{4}-\sin \frac{\pi(1-x)}{4}=-2 \sin \frac{\pi}{8} \cos \frac{\pi(1-2 x)}{8} \geq-2 \sin \frac{\pi}{8},
$$

SO

$$
\frac{F(x)}{\gamma}=\sum_{n=1}^{\infty} \gamma^{n-2} P_{n} \geq \frac{2 \sin (\pi x)}{\gamma}+\sqrt{2} \sin \left(\frac{\pi x}{2}+\frac{\pi}{4}\right)-2 \gamma \sin \frac{\pi}{8}-\frac{2 \gamma^{2}}{1-\gamma}
$$

The right hand side is increasing in $x \in[2 / 5,1 / 2]$ and decreasing in $\gamma$. Thus for $x \in[2 / 5,1 / 2]$,

$$
\frac{F(x)}{\gamma} \geq 2 \sqrt{2} \sin \frac{2 \pi}{5}+\sqrt{2} \sin \frac{9 \pi}{20}-\sqrt{2} \sin \frac{\pi}{8}-2-\sqrt{2}>0 .
$$

(iv) Assume $\left(u_{1}, u_{2}\right)=(0,1),\left(v_{1}, v_{2}\right)=(1,1)$. By Lemma 5.3. we only need to show that $(01) \chi_{x}(11)$ for $x \in[1 / 3,2 / 5]$. Then

$$
P_{2}=\cos \frac{\pi x}{2}-\sin \frac{\pi x}{2} \text { and } Q_{2}=-\sin \frac{\pi x}{2}-\cos \frac{\pi x}{2} \text {. }
$$

Put

$$
\begin{gathered}
p(x)=\frac{P_{1}}{\gamma}+P_{2}=\frac{2 \sin (\pi x)}{\gamma}+\left(\cos \frac{\pi x}{2}-\sin \frac{\pi x}{2}\right), \\
q(x)=Q_{1}+\frac{\gamma}{2} Q_{2}=2 \cos (\pi x)-\frac{\gamma}{2}\left(\sin \frac{\pi x}{2}+\cos \frac{\pi x}{2}\right) .
\end{gathered}
$$


Since $q(x)$ is decreasing in $[0,1 / 2]$, we have

$$
q(x) \geq q(2 / 5)>\frac{\sqrt{5}-1}{2}-\frac{\gamma}{\sqrt{2}} \geq \frac{\sqrt{5}-2}{2} .
$$

for each $0 \leq x \leq 2 / 5$. Since $p^{\prime}=\pi q / \gamma>0$, for each $x \in[1 / 3,2 / 5]$, we have

$$
p(x) \geq p(1 / 3) \geq \frac{\sqrt{3}}{\gamma}+\frac{\sqrt{3}-1}{2} \geq \sqrt{6}+\frac{\sqrt{3}-1}{2}>2.81 .
$$

Case 1. Assume also $v_{3}=0$. Then

$$
Q_{3}= \pm \sin \frac{\pi x}{4}+\cos \frac{\pi(1-x)}{4} \geq \cos \frac{\pi(1-x)}{4}-\sin \frac{\pi x}{4} .
$$

Since the right hand side is decreasing in $[0,1 / 2]$, we obtain

$$
Q_{3} \geq \cos \frac{\pi}{8}-\sin \frac{\pi}{8}>\frac{1}{2}
$$

and

$$
G(x) \geq Q_{1}+\frac{\gamma}{2} Q_{2}+\frac{\gamma^{2}}{4} Q_{3}-\frac{\gamma^{3}}{4-2 \gamma}>q(x)+\frac{\gamma^{2}}{8}-\frac{\gamma^{3}}{4-2 \gamma}
$$

Thus

$$
\frac{G(x)}{\gamma^{2}} \geq \frac{\sqrt{5}-2}{2 \gamma^{2}}+\frac{1}{8}-\frac{\gamma}{4-2 \gamma} \geq \sqrt{5}-2+\frac{1}{8}-\frac{1}{4 \sqrt{2}-2}>0 .
$$

Case 2. Assume $u_{3}=v_{3}=1$. Then

$$
P_{3}=\cos \frac{\pi(1+x)}{4}-\cos \frac{\pi x}{4}=-2 \sin \frac{\pi(1+2 x)}{8} \sin \frac{\pi}{8}>-\sqrt{2} \sin \frac{\pi}{8}
$$

and

$$
Q_{3}=\sin \frac{\pi x}{4}-\sin \frac{\pi(1+x)}{4}=-2 \sin \frac{\pi}{8} \cos \frac{\pi(1+2 x)}{8}>-\sin \frac{\pi}{4}=-\frac{\sqrt{2}}{2} .
$$

Subcase 2.1. $u_{4}=1$ and $v_{4}=0$. Then

$$
Q_{4}=\cos \frac{\pi(2-x)}{8}+\cos \frac{\pi(1-x)}{8}>\sqrt{2}>\frac{2 \gamma}{2-\gamma} .
$$

Therefore, for $x \in[0,2 / 5]$,

$$
\begin{aligned}
G(x) & =Q_{1}+\frac{\gamma}{2} Q_{2}+\frac{\gamma^{2}}{4} Q_{3}+\frac{\gamma^{3}}{8} Q_{4}-\frac{\gamma^{4}}{8-4 \gamma} \\
& \geq q(x)+\frac{\gamma^{2}}{4} Q_{3}+\frac{\gamma^{3}}{8}\left(Q_{4}-\frac{2 \gamma}{2-\gamma}\right) \\
& >q(x)-\frac{\sqrt{2}}{16}>0,
\end{aligned}
$$

where the last inequality follows from (5.3).

Subcase 2.2. $u_{4}=0$. Then

$$
P_{4} \geq \sin \frac{\pi(2-x)}{8}-\sin \frac{\pi(1-x)}{8}>0 .
$$


Thus

$$
\begin{array}{rlrl}
\frac{F(x)}{\gamma} & \geq \frac{P_{1}}{\gamma}+P_{2}+\gamma P_{3}+\gamma^{2} P_{4}-\frac{2 \gamma^{3}}{1-\gamma} & >p(x)+\gamma P_{3}-\frac{2 \gamma^{3}}{1-\gamma} \\
& \geq p(x)-\sqrt{2} \gamma \sin \frac{\pi}{8}-\frac{2 \gamma^{3}}{1-\gamma} & & \geq p(x)-\sin \frac{\pi}{8}-\sqrt{2}-1>0.01,
\end{array}
$$

where the last inequality follows from (5.4).

Subcase 2.3. $u_{4}=v_{4}=1$. Then

$$
P_{4}=\sin \frac{\pi(1-x)}{8}-\sin \frac{\pi(2-x)}{8}=-2 \sin \frac{\pi}{16} \cos \frac{\pi(3-2 x)}{16}>-2 \sin \frac{\pi}{16} ;
$$

and

$$
P_{5} \geq-\sin \frac{\pi(2-x)}{16}-\sin \frac{\pi(1-x)}{16}=-2 \sin \frac{\pi(3-2 x)}{32} \cos \frac{\pi}{32}>-2 \sin \frac{3 \pi}{32} .
$$

Therefore,

$$
\begin{aligned}
\frac{F(x)}{\gamma} & \geq p(x)+\gamma P_{3}+\gamma^{2} P_{4}+\gamma^{3} P_{5}-\frac{2 \gamma^{4}}{1-\gamma} \\
& \geq p(x)-\gamma \sqrt{2} \sin \frac{\pi}{8}-2 \gamma^{2} \sin \frac{\pi}{16}-2 \gamma^{3} \sin \frac{3 \pi}{32}-\frac{2 \gamma^{4}}{1-\gamma} \\
& \geq p(x)-\sin \frac{\pi}{8}-\sin \frac{\pi}{16}-\frac{1}{\sqrt{2}} \sin \frac{3 \pi}{32}-1-\frac{\sqrt{2}}{2}>0
\end{aligned}
$$

where the last inequality follows from (5.4).

Case 3. Assume $u_{3}=0$ and $v_{3}=1$. Then for $x \in[1 / 3,2 / 5]$,

$$
P_{3}=\cos \frac{\pi x}{4}+\cos \frac{\pi(1+x)}{4}=2 \cos \frac{\pi}{8} \cos \frac{\pi(1+2 x)}{8}>\sqrt{2} \cos \frac{\pi}{8} .
$$

Thus

$$
\begin{aligned}
\frac{1}{\gamma^{2}} F(x) \geq \frac{1}{\gamma^{2}} P_{1}+\frac{P_{2}}{\gamma}+P_{3}-\frac{2 \gamma}{1-\gamma} & >\frac{p(x)}{\gamma}+\sqrt{2} \cos \frac{\pi}{8}-\frac{2 \gamma}{1-\gamma} \\
& \geq \sqrt{2}\left(p(x)+\cos \frac{\pi}{8}-2-\sqrt{2}\right)>0,
\end{aligned}
$$

where the last inequality follows from (5.4).

(v) It follows from (i) (ii) and (iv).

(vi) It suffices to show that $0 \chi_{x(1)} 1$ for $x \in[1 / 5,1 / 2]$. But $x(1) \in[3 / 5,1)$, so the statement follows from (v) and Lemma 2.9.

Summarizing the estimates given by Lemma 5.3 and Lemma 5.6, we have

Lemma 5.7. Assume $\gamma \leq \sqrt{2} / 2$. Then

(1) For $x \in[1 / 5,2 / 5], e(2, x)=1$;

(2) For $x \in[0,1 / 5)$, the only possible non-trivial pairs in $E(2, x)$ are $(10,11)$ and $(11,10)$;

(3) For $x \in(2 / 5,1 / 2]$, the only possible non-trivial pairs in $E(2, x)$ are $(01,10),(01,11),(10,01)$ and $(11,01)$.

Proof. By Lemma $5.3(00) \varkappa_{x}(01)$ for all $x \in[0,1 / 2]$, since $0 \nsim_{x(0)} 1$. By Lemma 5.6 (i) and (iii), we have (00) $\chi_{x}(10)$ and (00) $\chi_{x}$ (11) for all $x \in$ $[0,1 / 2]$. For $x \in[2 / 5,1 / 2]$, by Lemma 5.6$]$ (vi), we also have (10) $\chi_{x}(11)$. Thus (3) holds. For $x \in[0,2 / 5]$, by Lemma 5.6 (ii) and (iv), (01) $\chi_{x}(10)$, 
(01) $\varkappa_{x}(11)$, so the only possible non-trivial pairs in $E(2, x)$ are $(10,11)$ and $(11,10)$. So $(2)$ holds. If $x \in[1 / 5,2 / 5]$, then $(10) \chi_{x}$ (11) by Lemma 5.6 (vi). Therefore (1) holds.

Proof of Proposition 5.4. We shall apply Lemma 2.12. Let $K_{0}=[1 / 5,2 / 5] \cup$ $[3 / 5,4 / 5], K_{1}=[0,1 / 5) \cup(4 / 5,1)$ and $K_{2}=(2 / 5,3 / 5)$. By Lemma 5.7, Proposition 2.2 (3) and Lemma [2.9] the conditions in Lemma 2.12 are satisfied with $q=2$ and with suitable choice of $(\varepsilon, \delta)$. Indeed,

- For $x \in[1 / 5,2 / 5]$, by Lemma [5.7, $e(2, x)=1$, so $e(2, x ; \varepsilon, \delta)=1$ for suitable choice of $\varepsilon, \delta$. By Lemma 2.9. this also holds for $x \in[3 / 5,4 / 5]$.

- For $x \in[0,1 / 5)$, take $\mathbf{a}_{x}=(10)$ and $\mathbf{b}_{x}=(11)$. Then $x(10), x(11) \in$ $K_{0}$. By Lemma [5.7. $(10,11)$ and $(11,10)$ are the only elements in $E(2, x)$. So by Proposition 2.2 (3) and Lemma 2.9, the condition (ii) in Lemma 2.12 is satisfied.

- For $x \in(2 / 5,1 / 2]$, let $\mathbf{a}_{x}=(01), \mathbf{b}_{x}=(10)$ and $\mathbf{c}_{x}=(11)$. Then $x(01), x(10) \in K_{0}$ and $x(11) \in K_{1}$; and by Lemma 5.7. $(01,10)$, $(01,11),(10,01)$ and $(11,01)$ are the only non-trivial pairs in $E(2, x)$. So by Proposition 2.2 (3) and Lemma2.9, the condition (iii) in Lemma 2.12 is satisfied.

Thus $\sigma(2)<1.61$.

\subsection{Step 3. When $\gamma^{2}>\sqrt{2} / 4$.}

Proposition 5.8. Assume $\gamma \leq 0.64$. Then $\sigma(2) \leq \sqrt{2}$.

An immediate corollary of this proposition and Corollary 5.5 is the following:

Corollary 5.9. If $\gamma^{2}>\sqrt{2} / 4$, then either $\sigma(1)<2 \gamma$ or $\sigma(2)<(2 \gamma)^{2}$.

The proof of Proposition 5.8 relies on the following estimates.

Lemma 5.10. If $\gamma \leq 0.64$, then for any $x \in[0,1 / 2]$, (01) $\nsim_{x}(11)$.

Proof. The case $0 \leq x \leq 2 / 5$ was treated in Lemma 5.6. Here we consider the case $x \in[2 / 5,1 / 2]$. Let $\mathbf{u}=\left\{u_{n}\right\}_{n=1}^{\infty}$ and $\mathbf{v}=\left\{v_{n}\right\}_{n=1}^{\infty}$ be such that $\left(u_{1} u_{2}\right)=(01)$ and $\left(v_{1} v_{2}\right)=(11)$. Let $F(x)=-(2 \pi)^{-1}(S(x, \mathbf{u})-S(x, \mathbf{v}))$ and let $P_{n}, Q_{n}$ be defined as above. Then

$$
P_{1}=2 \sin (\pi x) \geq 2 \sin \frac{2 \pi}{5}=\frac{\sqrt{10+2 \sqrt{5}}}{2}>1.902,
$$

and

$$
P_{2}=\cos \frac{\pi x}{2}-\sin \frac{\pi x}{2}>0 .
$$

Case 1. $u_{3}=0$ or $v_{3}=1$. Then

$$
\begin{aligned}
P_{3}=(-1)^{u_{3}} \cos \frac{\pi x}{4}-(-1)^{v_{3}} \cos \frac{\pi(1+x)}{4} \geq \cos \frac{\pi(1+x)}{4}-\cos \frac{\pi x}{4} \\
\geq-2 \sin \frac{\pi}{8} \sin \frac{\pi(1+2 x)}{8}>-\sqrt{2} \sin \frac{\pi}{8},
\end{aligned}
$$

so

$$
\begin{aligned}
F(x) & \geq P_{1}+\gamma P_{2}+\gamma^{2} P_{3}-\frac{2 \gamma^{3}}{1-\gamma} \\
& >1.902-\sqrt{2} \cdot 0.64^{2} \sin \frac{\pi}{8}-\frac{2 \cdot 0.64^{3}}{1-0.64}>0 .
\end{aligned}
$$


Case 2. $u_{3}=1$ and $v_{3}=0$. Then

$$
\begin{aligned}
P_{3}=-\cos \frac{\pi(1+x)}{4}-\cos \frac{\pi x}{4}=-2 \cos \frac{\pi}{8} \cos \frac{\pi(1+2 x)}{8} \\
>-2 \cos ^{2} \frac{\pi}{8}=-1-\frac{\sqrt{2}}{2}>-1.708 .
\end{aligned}
$$

Subcase 2.1. $i_{4}=1$ and $j_{4}=0$. Then

$$
P_{4}=-\sin \frac{\pi(2-x)}{8}-\sin \frac{\pi(x+3)}{8}=-2 \sin \frac{5 \pi}{16} \cos \frac{\pi(1+2 x)}{16}>-2 \sin \frac{5 \pi}{16}>-1.663 .
$$

and

$$
P_{5} \geq-\sin \frac{\pi(2-x)}{16}-\sin \frac{\pi(x+3)}{16}=-2 \sin \frac{5 \pi}{32} \cos \frac{\pi(1+2 x)}{32}>-2 \sin \frac{5 \pi}{32}>-0.942 .
$$

Thus

$$
\begin{aligned}
& F(x) \geq P_{1}+\gamma P_{2}+\gamma^{2} P_{3}+\gamma^{3} P_{4}+\gamma^{4} P_{5}-\frac{2 \gamma^{5}}{1-\gamma} \\
& \geq 1.902-0.64^{2} \cdot 1.708-\cdot 0.64^{3} \cdot 1.663-0.942 \cdot 0.64^{4}-\frac{2 \cdot 0.64^{5}}{1-0.64}>0.011 .
\end{aligned}
$$

Subcase 2.2. Either $i_{4}=0$ or $j_{4}=1$. Then

$P_{4} \geq \sin \frac{\pi(2-x)}{8}-\sin \frac{\pi(3+x)}{8}=-2 \sin \frac{\pi(1+2 x)}{16} \cos \frac{5 \pi}{16}>-2 \sin \frac{\pi}{8} \cos \frac{5 \pi}{16}>-0.556$.

Thus

$$
\begin{aligned}
F(x) \geq P_{1}+\gamma P_{2} & +\gamma^{2} P_{3}+\gamma^{3} P_{4}-\frac{2 \gamma^{4}}{1-\gamma} \\
> & 1.902-0.64^{2} \cdot 1.708-0.64^{3} \cdot 0.556-\frac{2 \cdot 0.64^{4}}{1-0.64}>0.124 .
\end{aligned}
$$

Summarizing the results obtained in Lemma 5.7 and Lemma 5.10, we have

Lemma 5.11. Assume $\gamma \leq 0.64$. Then

(1) For $x \in[1 / 5,2 / 5], e(2, x)=1$;

(2) For $x \in[0,1 / 5)$, the only possible non-trivial pairs in $E(2, x)$ are $(10,11)$ and $(11,10)$;

(3) For $x \in(2 / 5,1 / 2]$, the only possible non-trivial pairs in $E(2, x)$ are $(01,10)$ and $(10,01)$.

Proof. This follows immediately from Lemma 5.7 and Lemma 5.10

Proof of Proposition 5.8. Let $K=[1 / 5,2 / 5] \cup[3 / 5,4 / 5]$. Then the conditions in Lemma 2.10 are satisfied with $q=2$ and suitable choice of $\varepsilon, \delta$. Indeed,

- By Lemma 5.11 for each $x \in[1 / 5,2 / 5]$, we have $e(2, x)=1$. So by Lemma 2.9 and Proposition 2.2 (3), condition (i) of Lemma 2.10 is satsified with suitable choices of $(\varepsilon, \delta)$;

- By Lemma 5.11, for each $x \in[0,1 / 5),(10,11)$ and $(11,10)$ are the only non-trivial pairs in $E(2, x)$, and it is easily checked $x(10), x(11) \in K$. For $x \in(2 / 5,1 / 2]$, by Lemma [5.11, $(01,10)$ and $(10,01)$ are the only non-trivial pairs in $E(2, x)$ and it is easily checked that $x(10), x(01) \in$ 
$K$. Thus by Lemma 2.9 and Proposition 2.2 (3), condition (ii) of Lemma 2.10 is satsified.

Thus $\sigma(2) \leq \sqrt{2}$.

5.4. When $\gamma^{3}>\sqrt{2} / 8$.

Proposition 5.12. Assume $\gamma^{2} \leq \sqrt{2} / 4$. Then $\sigma(3) \leq \sqrt{2}$.

An immediate corollary of this proposition and Corollary 5.9 is the following:

Corollary 5.13. If $\gamma^{3}>\sqrt{2} / 8$, then $\sigma(q)<(2 \gamma)^{q}$ for some $q \in\{1,2,3\}$.

Lemma 5.14. Assume $\gamma^{2} \leq \sqrt{2} / 4$. If $\left(01 u_{3}\right) \sim_{x}\left(10 v_{3}\right)$ for some $x \in[0,1 / 2]$ then $x \in(17 / 36,1 / 2], u_{3}=1$ and $v_{3}=0$.

Proof. Note that $\gamma<0.6$. Let $\mathbf{u}=01 u_{3} \cdots, \mathbf{v}=10 v_{3} \cdots$ and let $F(x)=$ $-(2 \pi)^{-1}(S(x, \mathbf{u})-S(x, \mathbf{v})), G(x)=F^{\prime}(x) / \pi$. We continue to use the notation $x_{n}, y_{n}, P_{n}$ and $Q_{n}$.

If $x \leq 0.45$, then as in the proof of Lemma 5.3 .

$$
\begin{aligned}
G(x) & \geq 2 \cos (\pi x)-\frac{\gamma}{\sqrt{2}} \cos \left(\frac{\pi x}{2}+\frac{\pi}{4}\right)-\frac{\gamma^{2}}{2-\gamma} \\
& \geq 2 \cos (0.45 \pi)-0.6 \cos (0.475 \pi) / \sqrt{2}-0.36 / 1.4>0.02
\end{aligned}
$$

Assume $\left(u_{3}, v_{3}\right) \neq(1,0)$. We shall show that for each $x \in[9 / 20,1 / 2]$, $F(x)>0$. Indeed, in this case, $P_{1}=2 \sin (\pi x), P_{2}=-\sin \frac{\pi x}{2}-\cos \frac{\pi x}{2}$ and

$$
\begin{array}{rlrl}
P_{3} & \geq \cos \frac{\pi(1-x)}{4}-\cos \frac{\pi x}{4} & & =-2 \sin \frac{\pi}{8} \sin \frac{\pi(1-2 x)}{8} \\
& \geq-2 \sin \frac{\pi}{8} \sin \frac{\pi}{40} & \geq-\frac{\pi^{2}}{160} .
\end{array}
$$

Thus

$$
\begin{aligned}
F(x) & \geq P_{1}+\gamma P_{2}+\gamma^{2} P_{3}-\frac{2 \gamma^{3}}{1-\gamma} \\
& \geq 2 \sin (\pi x)-\gamma\left(\cos \frac{\pi x}{2}+\sin \frac{\pi x}{2}\right)-\frac{\gamma^{2} \pi^{2}}{160}-\frac{2 \gamma^{3}}{1-\gamma} \\
& \geq 2 \sin (\pi x)-0.6\left(\cos \frac{\pi x}{2}+\sin \frac{\pi x}{2}\right)-0.023-1.08 .
\end{aligned}
$$

For $x \in[9 / 20,1 / 2]$, this give us

$$
F(x)>2 \sin \frac{9 \pi}{20}-0.6 \sqrt{2}-1.103>0.013 .
$$

Assume now $\left(u_{3}, v_{3}\right)=(1,0)$. We shall show that $G(x)>0$ for all $x \in$ $[9 / 20,17 / 36]$. Indeed, in this case,

$$
Q_{3}=\cos \frac{\pi(2-x)}{4}-\cos \frac{\pi(1+x)}{4}=-2 \sin \frac{3 \pi}{8} \sin \frac{\pi(1-2 x)}{8}>-\frac{\pi}{40} .
$$


Thus

$$
\begin{aligned}
G(x) & =Q_{1}+\frac{\gamma}{2} Q_{2}+\frac{\gamma^{2}}{4} Q_{3}-\frac{\gamma^{3}}{2-\gamma} \\
& \geq 2 \cos (\pi x)-\frac{\gamma}{\sqrt{2}} \cos \left(\frac{\pi x}{2}+\frac{\pi}{4}\right)-\frac{\gamma^{2}}{4} \frac{\pi}{40}-\frac{\gamma^{3}}{4-2 \gamma} \\
& \geq 2 \cos \frac{17 \pi}{36}-\frac{0.6}{\sqrt{2}} \cos \frac{35 \pi}{72}-0.01-0.08 \\
& >0.174-0.02-0.01-0.08>0 .
\end{aligned}
$$

Lemma 5.15. Assume $\gamma<0.6$. If $x \in[0,1 / 2]$ and $\boldsymbol{u}, \boldsymbol{v}$ are distinct elements in $\mathcal{A}^{3}$ such that $(\boldsymbol{u}, \boldsymbol{v}) \in E(3, x)$, then either

$$
x \in[0,1 / 9) \text { and }(\boldsymbol{u}, \boldsymbol{v}) \in\{(101,110),(110,101),(010,011),(011,010)\} ;
$$

or

$$
x \in(17 / 36,1 / 2] \text { and }(\boldsymbol{u}, \boldsymbol{v}) \in\{(011,100),(100,011)\} .
$$

Proof. Lemma 5.14 particularly implies that $(01) \chi_{x}(10)$ for $x \in[2 / 5,17 / 36]$. Together with Lemma 5.11, it follows that

(a) $0 \nsim_{x} 1$ for $x \in[0,17 / 36]$.

By Lemma 2.9.

(a') $0 \nsim_{x} 1$ for $x \in[19 / 36,1]$.

Therefore, for $x \in[1 / 18,1 / 5],(10) \varkappa_{x}(11)$, since $x(1) \in[19 / 36,3 / 5]$. Together with Lemma 5.11, we obtain

(b) For $x \in[1 / 18,17 / 36], e(2, x)=1$;

(c) For $x \in[0,1 / 18)$, the only possible non-trivial pairs in $E(2, x)$ are $(10,11)$ or $(11,10)$;

(d) For $x \in(17 / 36,1 / 2]$, the only possible non-trivial pairs in $E(2, x)$ are $(01,10)$ or $(10,01)$.

Consider $x \in[1 / 9,17 / 36]$. If $\left(u_{1} u_{2} u_{3}\right) \sim_{x}\left(v_{1} v_{2} v_{3}\right)$ then by (b), $u_{1} u_{2}=v_{1} v_{2}$. Note that for any $u_{1} u_{2}, x\left(u_{1} u_{2}\right) \notin[17 / 36,19 / 36]$. Thus by (a) (and (a')), we have $u_{3}=v_{3}$. This proves that $e(3, x)=1$.

Consider $x \in[0,1 / 9)$ and $\left(u_{1} u_{2} u_{3}\right) \sim_{x}\left(v_{1} v_{2} v_{3}\right)$. Then by (a), $u_{1}=$ $v_{1}$. If $u_{1}=0$ then $x\left(u_{1}\right) \in[0,1 / 18)$, and $\left(u_{2} u_{3}\right) \sim_{x\left(u_{1}\right)}\left(v_{2} v_{3}\right)$, so by $(\mathrm{c})$, $\left(u_{2} u_{3}, v_{2} v_{3}\right) \in\{(10,11),(11,10)\}$. If $u_{1}=1$, then $x\left(u_{1}\right) \in[1 / 2,10 / 18]$, and $\left(u_{2} u_{3}\right) \sim_{x\left(u_{1}\right)}\left(v_{2} v_{3}\right)$, so by Lemma $5.11(3),\left(u_{2} u_{3}, v_{2} v_{3}\right) \in\{(01,10),(10,01)\}$.

Consider $x \in[17 / 36,1 / 2]$. Then by (d) and Lemma [5.14 the only possible non-trivial pairs in $E(3, x)$ are $(011,100)$ and $(100,011)$.

Proof of Proposition 5.12. Let $K=[1 / 9,17 / 36] \cup[36 / 19,8 / 9]$. The conditions in Lemma 2.10 are satisfied with $q=3$ and suitable choices of $(\varepsilon, \delta)$. Indeed, putting $L_{1}=(17 / 36,19 / 36), L_{2}=[0,1 / 9) \cup(8 / 9,1)$. by Lemma 5.15, Lemma 2.9 and Proposition 2.2 (3), there exist $\varepsilon>0$ and $\delta>0$ such that

- $e(3, x ; \varepsilon, \delta)=1$ for all $x \in K$.

- For $x \in L_{1}, e(3, x ; \varepsilon, \delta) \leq 2$ and the only non-trivial elements of $\mathcal{A}^{3}$ which appears in a non-trivial pair of $E(3, x)$ are (011) and (100) for which we have $x(011), x(100) \in K$. 
- For $x \in[0,1 / 9), e(3, x ; \varepsilon, \delta) \leq 2$ and the only elements of $\mathcal{A}^{3}$ which appear in non-trivial pairs of $E(3, x)$ are (010), (011), (101) and (110) for which $x(010), x(011), x(101), x(110) \in K$. By Lemma 2.9, similar properties hold for $x \in(8 / 9,1)$.

By Lemma 2.10, we have $\sigma(3) \leq \sqrt{2}$.

5.5. Last Step: When $\gamma^{3} \leq \sqrt{2} / 8$.

Proposition 5.16. Assume $\gamma^{3} \leq \sqrt{2} / 8$. Then $e(1)=1$.

Proof. It suffices to show that

$$
\text { (011) } \not_{x}(100) \text { for } x \in[17 / 36,1 / 2] \text {. }
$$

Indeed, by Lemma [5.15, it follows that $0 \chi_{x} 1$ for all $x \in[0,1 / 2]$. By Lemma 2.9. $0 \nsim_{x} 1$ also holds for $x \in[1 / 2,1]$.

To prove (5.5), let $\mathbf{u}=(011 \cdots), \mathbf{v}=(100 \cdots)$ and $F(x)=-(2 \pi)^{-1}(S(x, \mathbf{u})-$ $S(x, \mathbf{v}))$. We shall prove that $F(x)>0$ holds for each $x \in[17 / 36,1 / 2]$. We shall continue to use the notation $x_{n}, y_{n}, P_{n}$ and $Q_{n}$ as above. Then $P_{1}=2 \sin (\pi x)$, $P_{2}=-\sin \frac{\pi x}{2}-\cos \frac{\pi x}{2}$ and

$$
P_{3}=-\sin \frac{\pi(2-x)}{4}-\sin \frac{\pi(1+x)}{4}=-2 \sin \frac{3 \pi}{8} \cos \frac{\pi(1-2 x)}{8} .
$$

Put

$$
g(x)=P_{1}+\gamma P_{2}+\gamma^{2} P_{3} .
$$

Using $\gamma \leq 0.562$, it is easy to check that $g^{\prime \prime}<0$ on $[9 / 20,1 / 2]$. By calculation,

$$
g(9 / 20)>0.602 \text { and } g(1 / 2)>0.624 \text {. }
$$

It follows that

$$
g(x)>0.602 \text { for any } x \in[17 / 36,1 / 2] .
$$

Case 1. $i_{4}=0$ or $j_{4}=1$. Then

$P_{4} \geq-\left(\sin \frac{\pi(2-x)}{8}-\sin \frac{\pi(1+x)}{8}\right) \geq-2 \sin \frac{\pi(1-2 x)}{8} \geq-2 \sin \frac{\pi}{144}>-0.05$.

Then

$$
\begin{aligned}
F(x) & \geq g(x)+\gamma^{3} P_{4}-\frac{2 \gamma^{4}}{1-\gamma} \\
& >0.602-0.05 \gamma^{3}-2 \gamma^{4} /(1-\gamma)>0 .
\end{aligned}
$$

Case 2. $i_{4}=1$ and $j_{4}=0$. Then

$$
P_{4}=-\left(\sin \frac{\pi(2-x)}{8}+\sin \frac{\pi(1+x)}{8}\right) \geq-2 \sin \frac{3 \pi}{16}>-1.112
$$

and

$$
P_{5} \geq-\left(\sin \frac{\pi(2-x)}{16}+\sin \frac{\pi(1+x)}{16}\right) \geq-2 \sin \frac{3 \pi}{32}>-0.581
$$

Thus

$$
F(x) \geq g(x)+\gamma^{3} P_{4}+\gamma^{4} P_{5}-\frac{2 \gamma^{5}}{1-\gamma}>0.602-1.112 \gamma^{3}-0.581 \gamma^{4}-\frac{2 \gamma^{5}}{1-\gamma}>0.09 .
$$




\section{Appendix: A proof of Ledrappier's theorem}

This appendix is devoted to a proof of Ledrappier's theorem under a further assumption that $\phi^{\prime}$ is continuous (for simplicity). The proof is motivated by the original proof given in [10] and also the recent paper [9].

Let $b \geq 2$ be an integer and $\lambda \in(1 / b, 1)$ and let $f(x)=\sum_{n=0}^{\infty} \lambda^{n} \phi\left(b^{n} x\right)$. We use $z$ to denote a point in $\mathbb{R}^{2}$ and $B(z, r)$ denote the open ball in $\mathbb{R}^{2}$ centered at $z$ and of radius $r$. We assume that $\operatorname{dim}\left(m_{x}\right)=1$ holds for Lebesgue a.e. $x \in[0,1)$, which means for Lebesgue a.e. $x \in[0,1)$ and $\mathbb{P}$-a.e. $\mathbf{u} \in \mathcal{A}^{\mathbb{Z}^{+}}$,

$$
\lim _{r \rightarrow 0} \frac{\log \mathbb{P}(\{\mathbf{v}:|S(x, \mathbf{u})-S(x, \mathbf{v})| \leq r\})}{\log r}=1 .
$$

Let $\mu$ be the pushforward of the Lebesgue measure on $[0,1)$ under the map $x \mapsto(x, f(x))$. Let

and

$$
\underline{d}(\mu, z)=\liminf _{r \rightarrow 0} \frac{\log \mu(B(z, r))}{\log r}
$$

$$
\underline{D}=\operatorname{essinf} \underline{d}(\mu, z) .
$$

We shall prove that

$$
\underline{D} \geq D=2+\frac{\log \lambda}{\log b}
$$

This is enough to conclude that the Hausdorff dimension of the graph of $f$ is $D$. Indeed, by the mass distribution principle, it implies that the Hausdorff dimension is at least $D$. On the other hand, it is easy to check that $f$ is a $C^{2-D}$ function which implies that the Hausdorff dimension is at most $D$ (see for example Theorem 8.1 of [5]).

6.1. Telescope. Define $\Phi:[0,1) \times \mathbb{R} \rightarrow[0,1) \times \mathbb{R}$ as

$$
\Phi(x, y)=(b x \bmod 1,(y-\phi(x)) / \lambda) .
$$

Define $G:[0,1) \times \mathbb{R} \times \mathcal{A}^{\mathbb{Z}^{+}} \rightarrow[0,1) \times \mathcal{A}^{\mathbb{Z}^{+}}$as

$$
G(x, y, \mathbf{u})=\left(\Phi(x, y), u_{0} \mathbf{u}\right), \text { if } b x \in\left[u_{0}, u_{0}+1\right) .
$$

The graph of $f$ is an invariant repeller of the expanding map $\Phi$. We shall use neighborhoods bounded by unstable manifolds. For each $z_{0}=\left(x_{0}, y_{0}\right) \in \mathbb{R}^{2}$ and $\mathbf{u} \in \mathcal{A}^{\mathbb{Z}^{+}}$, let $\ell_{z_{0}, \mathbf{u}}(x)$ denote the unique solution of the initial value problem:

$$
y^{\prime}=-\gamma S(x, \mathbf{u}), y\left(x_{0}\right)=y_{0} .
$$

These curves are strong unstable manifolds of $\Phi$ and they satisfy the following property: for $z=(x, y), z_{0}=\left(x_{0}, y_{0}\right) \in\left[u_{0} / b,\left(u_{0}+1\right) / b\right) \times \mathbb{R}, u_{0} \in \mathcal{A}$,

$$
\Phi\left(x, \ell_{z_{0}, \mathbf{u}}(x)\right)=\left(b x-u_{0}, \ell_{\Phi\left(z_{0}\right), u_{0} \mathbf{u}}\left(b x-u_{0}\right)\right) .
$$

For $z_{0}=\left(x_{0}, y_{0}\right) \in[0,1) \times \mathbb{R}, \mathbf{u} \in \mathcal{A}^{\mathbb{Z}^{+}}$and $\delta_{1}, \delta_{2}>0$, let

$$
Q\left(z_{0}, \mathbf{u}, \delta_{1}, \delta_{2}\right)=\left\{(x, y): x \in[0,1),\left|x-x_{0}\right| \leq \delta_{1},\left|y-\ell_{z_{0}, \mathbf{u}}(x)\right| \leq \delta_{2}\right\} .
$$

The following observation was taken from [9].

Lemma 6.1 (Telescope). Let $\left\{\left(z_{i}, \boldsymbol{u}_{i}\right)\right\}_{i=0}^{n}$ be a $G$-orbit and let $x_{i}$ denote the first coordinate of $z_{i}$. For any $\delta_{1}, \delta_{2}>0$, if $\delta_{1} \leq x_{n}<1-\delta_{1}$, then

$$
\mu\left(Q\left(z_{0}, \boldsymbol{u}, \delta_{1} b^{-n}, \delta_{2} \lambda^{n}\right)\right)=b^{-n} \mu\left(Q\left(z_{n}, \boldsymbol{u}_{n}, \delta_{1}, \delta_{2}\right)\right) .
$$


Proof. Let $J_{i}=\left[x_{i}-\delta_{1} b^{i-n}, x_{i}+\delta_{1} b^{i-n}\right], Q_{i}=Q\left(z_{i}, \mathbf{u}_{i}, \delta_{1} b^{i-n}, \delta_{2} \lambda^{n-i}\right)$ and let $E_{i}=\left\{x \in J_{i}:(x, f(x)) \in Q_{i}\right\}$. Then $\mu\left(Q_{i}\right)=\left|E_{i}\right|$. Under the assumption $\delta_{1} \leq x_{n}<1-\delta_{1}, Q_{0}$ is mapped onto $Q_{n}$ diffemorphically under $\Phi^{n}$. Thus $J_{0}$ is mapped onto $J_{n}$ and $E_{0}$ is mapped onto $E_{n}$ diffeomorphically under the linear map $x \mapsto b^{n} x$. Thus $\left|E_{0}\right|=b^{-n}\left|E_{n}\right|$.

6.2. A version of Marstrand's estimate. Fix a constant $t \in(1 /(1+\alpha), 1)$.

Proposition 6.2. For $\mu \times \mathbb{P}$-a.e. $\left(z_{0}, \boldsymbol{u}\right)$,

$$
\liminf _{r \rightarrow 0} \frac{\log \mu\left(Q\left(z_{0}, \boldsymbol{u}, r^{t}, r\right)\right)}{\log r} \geq 1+t(\underline{D}-1) .
$$

Proof. It suffices to prove that for each $\xi>0$ and $\eta>0$, there is a subset $\Sigma$ of $[0,1) \times \mathbb{R} \times \mathcal{A}^{\mathbb{Z}^{+}}$with $(\mu \times \mathbb{P})(\Sigma)>1-\eta$ such that

$$
\liminf _{r \rightarrow 0} \frac{\log \mu\left(Q\left(z_{0}, \mathbf{u}, r^{t}, r\right)\right)}{\log r} \geq 1+t(\underline{D}-1)-3 \xi
$$

holds for all $\left(z_{0}, \mathbf{u}\right) \in \Sigma$. By Egoroff's theorem, we can choose $\Sigma$ with $(\mu \times$ $\mathbb{P})(\Sigma)>1-\eta$ for which there is $r_{0}>0$ such that for each $\left(z_{0}, \mathbf{u}\right) \in \Sigma$,

(S1) $\mathbb{P}\left(\left\{\mathbf{v}:\left|S\left(x_{0}, \mathbf{u}\right)-S\left(x_{0}, \mathbf{v}\right)\right| \leq r\right\}\right) \leq r^{1-\xi}$ for each $0<r \leq r_{0}$, where $x_{0}$ is the first coordinate of $z_{0}$;

(S2) $\mu\left(B\left(z_{0}, r\right)\right) \leq r \underline{D}-\xi$ for each $0<r \leq r_{0}$.

In the following we shall prove that for $r>0$ small enough,

$$
\int_{\mathbf{u}:\left(z_{0}, \mathbf{u}\right) \in \Sigma} \mu\left(Q\left(z_{0}, \mathbf{u}, r^{t}, r\right)\right) d \mathbb{P} \leq r^{1+t(\underline{D}-1)-2 \xi},
$$

holds for every $z_{0} \in[0,1) \times \mathbb{R}$. This is enough to conclude the proof. Indeed, let $\tau \in(0,1)$ be an arbitrary constant. Then by (6.3), there is $N$ such that for $n>N$,

$$
\mathbb{P}\left(\left\{\mathbf{u}:\left(z_{0}, \mathbf{u}\right) \in \Sigma, \mu\left(Q\left(z_{0}, \mathbf{u}, \tau^{n t}, \tau^{n}\right)\right)>\left(\tau^{n}\right)^{1+t(\underline{D}-1)-3 \xi}\right\}\right) \leq \tau^{n \xi}
$$

holds for every $z_{0} \in[0,1) \times \mathbb{R}$. By Fubini's theorem, this implies that

$$
\mu \times \mathbb{P}\left(\left\{\left(z_{0}, \mathbf{u}\right) \in \Sigma: \mu\left(Q\left(z_{0}, \mathbf{u}, \tau^{n t}, \tau^{n}\right)\right)>\left(\tau^{n}\right)^{1+t(\underline{D}-1)-3 \xi}\right\}\right) \leq \tau^{n \xi} .
$$

By Borel-Cantelli, it follows that for almost every $\left(z_{0}, \mathbf{u}\right) \in \Sigma, \mu\left(Q\left(z_{0}, \mathbf{u}, \tau^{n t}, \tau^{n}\right)\right) \leq$ $\left(\tau^{n}\right)^{1+t(\underline{D}-1)-3 \xi}$ holds for all $n$ large enough. The inequality (6.2) follows.

Let us now prove (6.3). We first prove

Claim. Provided that $r>0$ is small enough, for every $z_{0}, z \in[0,1) \times \mathbb{R}$, we have

$$
\mathbb{P}\left(\left\{\mathbf{u}:\left(z_{0}, \mathbf{u}\right) \in \Sigma, z \in Q\left(z_{0}, \mathbf{u}, r^{t}, r\right)\right\}\right) \leq C_{1}\left(\frac{r}{\left|z-z_{0}\right|}\right)^{1-2 \xi}
$$

where $C_{1}>0$ is a constant.

To prove this claim, let $z=(x, y), z_{0}=\left(x_{0}, y_{0}\right)$ and $h(x)=\ell_{z_{0}, \mathbf{u}}(x)$. Then $h(x)$ is $C^{1+\alpha}$ with uniformly bounded norm. So

$$
\begin{array}{r}
\left|y-y_{0}+\gamma S\left(x_{0}, \mathbf{u}\right)\left(x-x_{0}\right)\right| \leq|y-h(x)|+\left|h(x)-h\left(x_{0}\right)-h^{\prime}\left(x_{0}\right)\left(x-x_{0}\right)\right| \\
\leq r+\left|\int_{x_{0}}^{x}\left(h^{\prime}(s)-h^{\prime}\left(x_{0}\right)\right) d s\right| \leq r+C r^{t(1+\alpha)}<2 r,
\end{array}
$$


provided that $r$ is small enough. Thus

$$
\left\{S\left(x_{0}, \mathbf{u}\right):\left(z_{0}, \mathbf{u}\right) \in \Sigma, z \in Q\left(z_{0}, \mathbf{u}, r^{t}, r\right)\right\}
$$

is contained in an interval of length $2 r /\left(\gamma\left|x-x_{0}\right|\right)$. Since

$$
\left|z-z_{0}\right| \leq\left|x-x_{0}\right|+\left|y-y_{0}\right| \leq\left(1+\gamma\left|S\left(x_{0}, \mathbf{u}\right)\right|\right)\left|x-x_{0}\right|+2 r,
$$

and $|S(x, \mathbf{u})|$ is uniformly bounded, the inequality (6.4) follows from the property (S1). Note that if $2 r /\left(\gamma\left|x-x_{0}\right|\right)>r_{0}$, then $r /\left|z-z_{0}\right|$ is bounded away from zero, so (6.4) holds for sufficiently large $C_{1}$, since the left hand side of this inequality does not exceed one.

We continue the proof of (6.3). Note that there is a constant $C_{2}>0$ such that for every $r>0$ and any $z_{0} \in[0,1) \times \mathbb{R}$,

$$
\bigcup_{\mathbf{u} \in \mathcal{A}^{\mathbb{Z}^{+}}} Q\left(z_{0}, \mathbf{u}, r^{t}, r\right) \subset B\left(z_{0}, C_{2} r^{t}\right) .
$$

Of course we may assume there is $\mathbf{u}$ such that $\left(z_{0}, \mathbf{u}\right) \in \Sigma$. Thus for $R>0$ small enough, we may apply (S2) and obtain

$$
\begin{aligned}
\int_{B\left(z_{0}, R\right)} \frac{d \mu(z)}{\left|z-z_{0}\right|^{1-2 \xi}} & =\sum_{n=0}^{\infty} \int_{e^{-n-1} R \leq\left|z-z_{0}\right|<e^{-n} R} \frac{d \mu(z)}{\left|z-z_{0}\right|^{1-2 \xi}} \\
& \leq \sum_{n=0}^{\infty} \frac{\mu\left(B\left(z_{0}, e^{-n} R\right)\right)}{\left(e^{-n-1} R\right)^{1-2 \xi}} \leq \sum_{n=0}^{\infty} \frac{\left(e^{-n} R\right)^{\underline{D}-\xi}}{\left(e^{-n-1} R\right)^{1-2 \xi}} \\
& =C(\xi) R^{\underline{D}-1+\xi},
\end{aligned}
$$

where $C(\xi)$ is a constant depending on $\xi$ and $\underline{D}$. By Fubini's theorem,

$$
\begin{aligned}
& \int_{\mathbf{u}:\left(z_{0}, \mathbf{u}\right) \in \Sigma} \mu\left(Q\left(z_{0}, \mathbf{u}, r^{t}, r\right) d \mathbb{P}(\mathbf{u})\right. \\
= & \int_{\mathbf{u}:\left(z_{0}, \mathbf{u}\right) \in \Sigma} \int_{\mathbb{R}^{2}} 1_{Q\left(z_{0}, \mathbf{u}, r^{t}, r\right)}(z) d \mu(z) d \mathbb{P}(\mathbf{u}) \\
= & \int_{B\left(z_{0}, C_{2} r^{t}\right)} \mathbb{P}\left(\left\{\mathbf{u}:\left(z_{0}, \mathbf{u}\right) \in \Sigma, z \in Q\left(z_{0}, \mathbf{u}, r^{t}, r\right)\right\}\right) d \mu(z) \\
& \leq C_{1} r^{1-2 \xi} \int_{B\left(z_{0}, C_{2} r^{t}\right)} \frac{d \mu(z)}{\left|z-z_{0}\right|^{1-2 \xi}} \\
& \leq C^{\prime} r^{1+t(\underline{D}-1)-(2-t) \xi}<r^{1+t(\underline{D}-1)-2 \xi},
\end{aligned}
$$

provided that $r$ is small enough.

We are ready to complete the proof of Ledrappier's theorem. For any $\xi>0$, $\eta>0$, by Proposition 6.2 and Egroff's theorem, we can pick up a subset $\Sigma$ of $\mathbb{R}^{2} \times \mathcal{A}^{\mathbb{Z}^{+}}$and a constant $r_{*}>0$ such that $(\mu \times \mathbb{P})(\Sigma)>1-3 \eta$ and such that for each $(z, \mathbf{u}) \in \Sigma$,

$$
\mu\left(Q\left(z, \mathbf{u}, r^{t}, r\right)\right) \leq r^{1+t(\underline{D}-1)-\xi} \text { for each } 0<r<r_{*} .
$$

We may further assume that $\Sigma \subset[\eta, 1-\eta] \times \mathbb{R} \times \mathcal{A}^{\mathbb{Z}^{+}}$.

Note that $\mu \times \mathbb{P}$ is an ergodic invariant measure for the map $G$. By Birkhorff's Ergodic Theorem, for almost every $\left(z_{0}, \mathbf{u}_{0}\right)$, there is an increasing sequence 
$\left\{n_{k}\right\}_{k=1}^{\infty}$ of positive integers such that $G^{n_{k}}\left(z_{0}, \mathbf{u}_{0}\right) \in \Sigma$ and

$$
\liminf _{k \rightarrow \infty} n_{k} / n_{k+1}>1-3 \eta \text {. }
$$

For each $n=1,2, \ldots$, put $\delta_{n}=\gamma^{n t /(1-t)} b^{-n}, r_{n}=\gamma^{n /(1-t)}$, so that

$$
r_{n}=\delta_{n} \lambda^{-n}, \text { and } r_{n}^{t}=\delta_{n} b^{n} .
$$

Let us prove that for $k$ sufficiently large,

$$
\frac{\log \mu\left(Q\left(z_{0}, \mathbf{u}_{0}, \delta_{n_{k}}, \delta_{n_{k}}\right)\right)}{\log \delta_{n_{k}}} \geq \underline{D}+(D-\underline{D}) A_{1}-A_{2} \xi,
$$

where $A_{1}, A_{2}$ are positive constants depending only on $\lambda$ and $b$.

Indeed, by Lemma 6.1, for $k$ large enough,

$$
\mu\left(Q\left(z_{0}, \mathbf{u}_{0}, \delta_{n_{k}}, \delta_{n_{k}}\right)\right)=\frac{\mu\left(Q\left(G^{n_{k}}\left(z_{0}, \mathbf{u}_{0}\right), r_{n_{k}}^{t}, r_{n_{k}}\right)\right)}{b^{n_{k}}} \leq \frac{r_{n_{k}}^{1+t(\underline{D}-1)-\xi}}{b^{n_{k}}} .
$$

Using definition of $r_{n}$ and $\delta_{n}$, this gives us

$$
\mu\left(Q\left(z_{0}, \mathbf{u}_{0}, \delta_{n_{k}}, \delta_{n_{k}}\right)\right) \leq \delta \frac{D}{n_{k}} \times\left(b^{-n_{k}}\right)^{D-\underline{D}} r_{n_{k}}^{-\xi},
$$

Thus (6.6) holds with $A_{1}=\log b /\left(\log b+t \log \gamma^{-1} /(1-t)\right)$ and $A_{2}=\log \gamma /(t \log \gamma+$ $\left.(1-t) \log b^{-1}\right)$.

By (6.5), for each $n$ large enough, there is $k$ such that $(1-3 \eta) n_{k}<n_{k-1}<$ $n \leq n_{k}$. It follows that

$$
\liminf _{n \rightarrow \infty} \frac{\log \mu\left(Q\left(z_{0}, \mathbf{u}_{0}, \delta_{n}, \delta_{n}\right)\right)}{\log \delta_{n}} \geq(1-3 \eta)\left(\underline{D}+(D-\underline{D}) A_{1}-A_{2} \xi\right) .
$$

Since $\ell_{x_{0}, \mathbf{u}_{0}}$ is a smooth curve, there exists $\kappa \in(0,1)$ such that $Q\left(z_{0}, \mathbf{u}_{0}, \delta_{k}, \delta_{k}\right)$ contains $B\left(z_{0}, \kappa \delta_{k}\right)$ for each $k$. Therefore,

$$
\underline{d}\left(\mu, z_{0}\right)=\liminf _{n \rightarrow \infty} \frac{\log \mu\left(Q\left(z_{0}, \mathbf{u}_{0}, \delta_{n}, \delta_{n}\right)\right)}{\log \delta_{n}} \geq(1-3 \eta)\left(\underline{D}+(D-\underline{D}) A_{1}-A_{2} \xi\right) .
$$

Since this estimate holds for $\mu$-a.e. $z_{0}$, we obtain

$$
\underline{D} \geq(1-3 \eta)\left(\underline{D}+A_{1}(D-\underline{D})-A_{2} \xi\right) .
$$

As $\xi, \eta$ can be chosen arbitrarily small, we conclude

$$
\underline{D} \geq \underline{D}+A_{1}(D-\underline{D}),
$$

which means $\underline{D} \geq D$, as desired.

\section{REFERENCES}

[1] R. Bamón, J. Kiwi, J. Rivera-Letelier and R. Urzúa. On the topology of solenoidal attaractors of the cylinder. Ann. I. Poincaré - AN 23 (2006), 209-236.

[2] K. Barański, B, Bárány and J. Romanowska. On the dimension of the graph of the classical Weierstrass function. Adv. Math. 265 (2014), 32-59.

[3] A.S. Besicovitch and H.D. Ursell. Sets of fractional dimensions (V): On dimensional numbers of some continuous curves. J. London Math. Soc. 1 (1937). No 1, 18-25.

[4] P. Erdös. On the smooth properties of a family of Bernoulli convolutions. Amer. J. Math. 62 (1940), 180-186.

[5] K. Falconer. The geometry of fractal sets Cambridge University Press, 1985.

[6] G. H. Hardy. Weierstrass's non-differentiable function. Trans. Amer. Math. Soc. 17 (1916), 301-325.

[7] T. Y. Hu and K.-S. Lau. Fractal dimensions and singularities of the Weierstrass type functions. Trans. Amer. Math. Soc. 335 (1993), 649-655. 
[8] J.L. Kaplan, J. Mallet-Paret and J.A. Yorke. The Lyapunov dimension of a nowhere differentiable attracting torus. Ergodic Theory Dynam. Systems. 4 (1984), 261-281.

[9] Gerhard Keller. An elementary proof for the dimension of the graph of the classical Weierstrass function. arXiv: 1406.3571v4.

[10] F. Ledrappier. On the dimension of some graphs. Contemp. Math 135 (1992), 285-293.

[11] F. Ledrappier and L.S. Young. The metric entropy of diffeomorphisms. II. Relations between entropy, exponents and dimension. Ann. of Math. 122 (1985), No. 3, 540-574.

[12] B. Mandelbrot. Fractals: form, chance, and dimension. W.H. Freeman and Co., San Francisco, 1977.

[13] Y. Peres and B. Solomyak. Absolute continuity of Bernoulli convolutions, a simple proof. Math. Research Letters, 3 (1996), 231-239.

[14] F. Rezakhanlou. The packing measure of the graphs and level sets of certain continuous functions. Math. Proc. Cambridge Philos. Soc. 104 (1988), 347-360.

[15] B. Solomyak. On the random series $\sum \pm \lambda^{n}$ (an Erdös problem). Ann. Math. 142 (1995), 611-625.

[16] Masato Tsujii. Fat Solenoidal attractors. Nonlinearity 14 (2001), No. 5, 1011-1027.

Shanghai Center for Mathematical Sciences, Fudan University, 220 Handan Road, Shanghai, China 200433

E-mail address: wxshen@fudan.edu.cn 